\title{
Stability and internal structure of vortices in spin-1 Bose-Einstein condensates with conserved magnetization
}

\author{
Justin Lovegrove, Magnus O. Borgh, and Janne Ruostekoski \\ Mathematical Sciences, University of Southampton, Southampton SO17 1BJ, United Kingdom
}

(Received 21 December 2015; published 18 March 2016)

\begin{abstract}
We demonstrate how conservation of longitudinal magnetization can have pronounced effects on both stability and structure of vortices in the atomic spin-1 Bose-Einstein condensate by providing a systematic characterization of nonsingular and singular vortex states. Constructing spinor wave functions for vortex states that continuously connect ferromagnetic and polar phases, we systematically derive analytic models for nonrotating cores of different singular vortices and for composite defect states with distinct small- and large-distance topology. We explain how the conservation law provides a stabilizing mechanism when the coreless vortex imprinted on the condensate relaxes in the polar regime of interatomic interactions. The resulting structure forms a composite defect: The inner ferromagnetic coreless vortex deforms toward an outer singly quantized polar vortex. We also numerically show how other even more complex hierarchies of vortex-core topologies may be stabilized. Moreover, we analyze the structure of the coreless vortex also in a ferromagnetic condensate and show how reducing magnetization leads to a displacement of the vortex from the trap center and eventually to the deformation and splitting of its core where a singular vortex becomes a lower-energy state. For the case of singular vortices, we find that the stability and the core structure are notably less influenced by the conservation of magnetization.
\end{abstract}

DOI: 10.1103/PhysRevA.93.033633

\section{INTRODUCTION}

The multicomponent order parameters of spinor BoseEinstein condensates (BECs), created in all-optical traps [1] that retain the atomic spin degree of freedom [2], provide a highly suitable laboratory for the study of topological defects and textures. The accelerating interest in the physics of defect structures in spinor BECs is illustrated by the very recent realization of point defects corresponding to analogs of Dirac magnetic monopoles [3] and 't Hooft-Polyakov monopoles [4]. In addition to the creation of defects, there is increasing interest in the structure of the defect cores as highlighted by the recent experimental observation of the splitting of singly quantized vortices into pairs of half-quantum vortices [5]. These recent experiments follow on experiments that have already demonstrated controlled preparation of nonsingular vortices and related textures [6-9] and out-of-equilibrium production of singular vortices in rapid phase transitions [10], as well as observations of spontaneously formed spin textures $[11,12]$. The experimental advances have been accompanied by a rapidly increasing body of theoretical studies of spinor BECs (see, e.g., Ref. [13] and references therein), describing, in particular, singular and nonsingular vortices and textures [14-34], as well as monopoles [35-39] and defect structures that cross a topological interface [40,41]. Complex topological textures are also possible in two-component (pseudospin- $\frac{1}{2}$ ) BECs [42-52].

When the energy of an optically trapped spinor BEC relaxes, its longitudinal magnetization is approximately conserved on time scales where $s$-wave scattering dominates the dynamics (over, e.g., weak dipolar interactions and scattering with high-temperature atoms; in the presence of resonant magnetic-field driving or spin-component phase separation, the importance of the dipole-dipole interactions can be enhanced) [53-56]. Here we provide a systematic characterization of the nonsingular and singular-vortex states in the spin-1 BEC and explain how conservation of magnetization can have pronounced effects on their stability and structure. These are determined by analytic models and numerically by relaxing the energy of suitable trial wave functions representing the defect states. To accurately account for conservation of magnetization, we explicitly impose the constraint throughout the energy-minimization procedure.

The spin-1 BEC exhibits two phases, ferromagnetic (FM) and polar, depending on the spin-dependent part of the interaction, each with a distinct family of vortices. In Ref. [34] we demonstrated that a FM coreless vortex phase imprinted on a spin-1 BEC with polar interactions [6] can be energetically stable as a composite topological defect when the conserved magnetization causes the phases to mix. The coreless vortex appears as the extended core region in a defect structure that continuously approaches a singly quantized polar vortex away from the vortex line. Here we extend these studies by explicitly constructing spinor wave functions for vortex states that interpolate smoothly between the FM and polar phases. From the resulting analytic models we systematically derive expressions for nonrotating cores of different singular vortices and a set of composite defect solutions with distinct smalland large-distance topology. We then suggest how precise control over the magnitude and spatial profile of the quadratic Zeeman shift can be used to stabilize also complex hierarchies of vortex-core topologies.

Our analytic models for the vortex spinor wave functions that simultaneously describe singular vortices and their nonrotating superfluid cores interpolate smoothly from the ground-state manifold to the orthogonal phase. Whereas a vortex in a scalar BEC always represents a depletion of the atomic density, such filled cores can appear in the spinor $\mathrm{BEC}$ as energy relaxes as a result of energetic competition in a hierarchy of characteristic length scales. The singularity of the order parameter is then accommodated by exciting the spinor wave function out of the ground-state manifold, becoming orthogonal to it at the singularity. In Ref. [26] we 
demonstrated how this mechanism leads to stable deformations of singly quantized vortices in both phases of the spin-1 BEC. We demonstrate here that these results are qualitatively robust when the relaxation model is refined to explicitly conserve magnetization as long as the magnetization is not too strong, including the recently observed [5] splitting of a singly quantized polar vortex into a pair of half-quantum vortices. Our analytic solutions then describe the relaxed vortex cores that could be observed in experiment.

Furthermore, we also provide a detailed analysis of a coreless vortex in the FM regime when the magnetization is explicitly conserved. We find that both the spin structure and the displacement of the coreless vortex are affected by the value of the magnetization: By displacing the vortex from the trap-rotation axis, the total angular momentum remains approximately constant for a range of magnetization strengths. From our numerical results, we explain the mechanism by which a coreless vortex becomes energetically unstable when magnetization is weak and gives way to a singular-vortex ground state, as we briefly noted in Ref. [34].

The article is organized as follows. In Secs. II and III we briefly review the mean-field theory of spin-1 BECs and outline the numerical magnetization-conserving energy-minimization method. Section IV gives an overview of the basic vortices supported by the two phases. We then construct analytic wave functions for vortex states that interpolate between the FM and the polar limits in Sec. V. Section VI investigates stability and structure of coreless vortices in both FM and polar interaction regimes. In Sec. VII we demonstrate the effects of a conserved weak magnetization on the core structure of singular vortices. We end by giving examples of how more complex core hierarchies can be stabilized in a spin-1 BEC in Sec. VIII before summarizing our findings in Sec. IX.

\section{SPIN-1 MEAN-FIELD THEORY}

We treat the spin-1 BEC in the Gross-Pitaevskii mean-field theory. The Hamiltonian density then reads $[13,57]$

$$
\begin{aligned}
\mathcal{H}= & \frac{\hbar^{2}}{2 m}|\nabla \Psi|^{2}+\frac{1}{2} m \omega^{2} r^{2} n+\frac{c_{0}}{2} n^{2}+\frac{c_{2}}{2} n^{2}|\langle\hat{\mathbf{F}}\rangle|^{2} \\
& +g_{1} n\langle\mathbf{B} \cdot \hat{\mathbf{F}}\rangle+g_{2} n\left\langle(\mathbf{B} \cdot \hat{\mathbf{F}})^{2}\right\rangle,
\end{aligned}
$$

where $n=\Psi^{\dagger} \Psi$ is the atom density and $m$ is the atomic mass. We have assumed an isotropic, harmonic trap of frequency $\omega$. The condensate wave function $\Psi$ is now a three-component spinor,

$$
\Psi(\mathbf{r})=\sqrt{n(\mathbf{r})} \zeta(\mathbf{r})=\sqrt{n(\mathbf{r})}\left(\begin{array}{c}
\zeta_{+}(\mathbf{r}) \\
\zeta_{0}(\mathbf{r}) \\
\zeta_{-}(\mathbf{r})
\end{array}\right), \quad \zeta^{\dagger} \zeta=1,
$$

in the basis of spin projection onto the $z$ axis. The local condensate spin is the expectation value $\langle\hat{\mathbf{F}}\rangle=\zeta_{\alpha}^{\dagger} \hat{\mathbf{F}}_{\alpha \beta} \zeta_{\beta}$ of the spin operator $\hat{\mathbf{F}}$, defined as a vector of spin-1 Pauli matrices. Linear and quadratic Zeeman shifts, of strength $g_{1}$ and $g_{2}$, respectively, described by the last two terms of Eq. (1), may arise from a weak external magnetic field $\mathbf{B}$.

Spin-independent and spin-dependent interaction terms with strengths $c_{0}=4 \pi \hbar^{2}\left(2 a_{2}+a_{0}\right) / 3 m$ and $c_{2}=4 \pi \hbar^{2}\left(a_{2}-\right.$ $\left.a_{0}\right) / 3 m$, respectively, arise from the two scattering channels of colliding spin-1 atoms with $s$-wave scattering lengths $a_{0}$ and $a_{2}$. Minimization of the interaction energy then leads to the two distinct phases of the spin-1 BEC: $c_{2}<0$ favors the $|\langle\hat{\mathbf{F}}\rangle|=1$ ferromagnetic (FM) phase (e.g., in ${ }^{87} \mathrm{Rb}$ ), while the $|\langle\hat{\mathbf{F}}\rangle|=0$ polar phase is favored when $c_{2}>0\left(\right.$ e.g., in $\left.{ }^{23} \mathrm{Na}\right)$.

We find stable vortex structures by minimizing the free energy $E=\int d^{3} r \mathcal{H}-\Omega\left\langle\hat{L}_{z}\right\rangle$ in the frame rotating at frequency $\Omega$ about the $z$ axis, using imaginary-time propagation of the coupled Gross-Pitaevskii equations. However, the only spinflip processes possible in $s$-wave scattering are $2|m=0\rangle \rightleftharpoons$ $|m=+1\rangle+|m=-1\rangle$. Therefore, $s$-wave interaction does not change the longitudinal magnetization,

$$
M=f_{+}-f_{-}=\frac{1}{N} \int d^{3} r n(\mathbf{r}) F_{z}(\mathbf{r}),
$$

where $f_{ \pm}=N_{ \pm} / N$. Here the total and the $|m= \pm 1\rangle$ level populations are denoted by $N$ and $N_{ \pm}$, respectively. We have also introduced the $z$ component of the $\operatorname{spin} F_{z}=\hat{\mathbf{z}} \cdot\langle\hat{\mathbf{F}}\rangle$.

Consequently, $M$ is approximately conserved on time scales where $s$-wave scattering dominates over, e.g., dipolar interactions and collisions with high-temperature atoms. This is the relevant time scale in present experiments with spinor BECs of alkali-metal atoms $[2,54,56]$. We take this conservation strictly into account throughout energy relaxation by simultaneously renormalizing $N$ and $M$ at each step of imaginary-time evolution.

The interaction terms in Eq. (1) give rise to the characteristic density and spin healing lengths,

$$
\xi_{n}=l\left(\frac{\hbar \omega}{2 c_{0} n}\right)^{1 / 2}, \quad \xi_{F}=l\left(\frac{\hbar \omega}{2\left|c_{2}\right| n}\right)^{1 / 2},
$$

where we have introduced the oscillator length $l=(\hbar / m \omega)^{1 / 2}$ of the harmonic confinement. The healing lengths determine, respectively, the length scales over which the atomic density $n(\mathbf{r})$ and the spin magnitude $|\langle\hat{\mathbf{F}}\rangle|$ heal around a local perturbation. When magnetization is not conserved, $\xi_{n}$ and $\xi_{F}$ determine the core size of singular defects $[26,36]$. If $\xi_{F}$ is sufficiently larger than $\xi_{n}$, it becomes energetically favorable to avoid depleting the atomic density, instead accommodating the singularity by exciting the wave function out of its ground-state manifold. The core then expands to the order of $\xi_{F}$, instead of the smaller $\xi_{n}$ that determines the size of a core with vanishing density. The lower gradient energy in the larger core offsets the cost in interaction energy.

Conservation of magnetization introduces a third length scale $\eta_{\mathrm{f}}$, which is the size required for a magnetized vortex core in an otherwise unmagnetized condensate to give rise to a given magnetization. In order to estimate the magnetization length scale, we represent the magnetized core by a cylinder of radius $\eta_{\mathrm{f}}$, with $\langle\hat{\mathbf{F}}\rangle=\hat{\mathbf{z}}$ everywhere inside the core and $|\langle\hat{\mathbf{F}}\rangle|=0$ outside. The total magnetization is then

$$
M\left(\eta_{\mathrm{f}}\right)=\frac{1}{N} \int d^{3} r \Theta\left(\eta_{\mathrm{f}}-\rho\right) n_{\mathrm{TF}}(\mathbf{r}),
$$

where $\rho=\left(x^{2}+y^{2}\right)^{1 / 2}$ and $\Theta$ is the Heaviside function. We approximate the atomic-density profile by the Thomas-Fermi solution

$$
n_{\mathrm{TF}}(r)=\frac{15 N}{8 \pi R_{\mathrm{TF}}^{3}}\left(1-\frac{r^{2}}{R_{\mathrm{TF}}^{2}}\right), \quad r \leqslant R_{\mathrm{TF}},
$$


where $r=\left(\rho^{2}+z^{2}\right)^{1 / 2}$, and

$$
R_{\mathrm{TF}}=l\left(\frac{15}{4 \pi} \frac{N c_{\mathrm{p}, \mathrm{f}}}{\hbar \omega l^{3}}\right)^{1 / 5}
$$

is the Thomas-Fermi radius. Here $c_{\mathrm{p}}=c_{0}$ in a BEC with polar interactions, and $c_{\mathrm{f}}=c_{0}+c_{2}$ in the FM regime. Computing the integral in Eq. (5) and solving for $\eta_{\mathrm{f}}$ as a function of $M$, we obtain

$$
\eta_{\mathrm{f}}=R_{\mathrm{TF}} \sqrt{1-(1-M)^{2 / 5}} .
$$

An analogous length scale $\eta_{\mathrm{p}}$ may be defined as the size required for an unmagnetized vortex core in an otherwise magnetized condensate to give rise to a given magnetization. We represent the unmagnetized core by a cylinder of radius $\eta_{\mathrm{p}}$, with $|\langle\hat{\mathbf{F}}\rangle|=0$ everywhere inside the core and $\langle\hat{\mathbf{F}}\rangle=\hat{\mathbf{z}}$ outside. The total magnetization may then be calculated, again by assuming a Thomas-Fermi density profile, as

$$
M\left(\eta_{\mathrm{p}}\right)=\frac{1}{N} \int d^{3} r \Theta\left(\rho-\eta_{\mathrm{p}}\right) n_{\mathrm{TF}}(\mathbf{r}) .
$$

Solving for $\eta_{\mathrm{p}}$ yields

$$
\eta_{\mathrm{p}}=R_{\mathrm{TF}} \sqrt{1-M^{2 / 5}}
$$

\section{NUMERICAL METHOD}

The energetic stability of vortex configurations, and the corresponding stable core structures, can be determined by numerically minimizing the free energy of suitable initial spinor wave functions in the rotating frame. Spinor wave functions representing specific vortices that can be used as such prototypes are constructed in Sec. IV. We minimize the energy by propagating the coupled Gross-Pitaevskii equations derived from Eq. (1) in imaginary time. Due to the renormalization of the wave function in each time step, the longitudinal magnetization $M$ [Eq. (3)] can change during the energy relaxation. However, the interaction terms in Eq. (1) arise from $s$-wave scattering, which preserves the relative populations of the $\zeta_{ \pm}$spinor components. Therefore, $M$ is approximately conserved on the time scales of interest here, where $s$-wave scattering dominates and we may neglect processes that cause magnetization to relax (e.g., due to dipolar interactions).

In order to conserve $M$ throughout the relaxation process, the imaginary-time propagation is performed using a split-step algorithm [58], where both the total number of atoms $N$ and the magnetization $M$ are constrained in each time step. This represents a different physical mechanism than that used in numerical techniques where an effective linear Zeeman term is used as a Lagrange multiplier for the magnetization of the final state, without explicitly conserving magnetization during relaxation $[14,16,17,29]$. With our method, the value of the conserved magnetization is determined by the initial state. We therefore construct initial wave functions from the appropriate spinors in Sec. IV by adjusting the relative populations of the components to yield the desired magnetization.

We consider a condensate in an isotropic, three-dimensional (3D), harmonic trap with interaction strengths chosen such that $N c_{0}=1000 \hbar \omega l^{3}$. The spin-dependent nonlinearity is kept fixed at $N c_{2}=-5 \hbar \omega l^{3}\left(N c_{2}=36 \hbar \omega l^{3}\right)$ in the FM (polar) regime, which is consistent with the experimentally measured ratio of $c_{2} / c_{0}$ for $F=1{ }^{87} \mathrm{Rb}\left({ }^{23} \mathrm{Na}\right)[59,60]$.

\section{BASIC VORTICES IN THE SPIN-1 BEC}

The set of physically distinguishable, energetically degenerate states defines the order-parameter manifold, the symmetry properties of which determine the families of vortices it supports. Here we give a brief overview of the basic spin-1 vortices in both phases before discussing the effects of magnetization in Secs. VI and VII. A more complete presentation can be found, for example, in Refs. [26,61].

\section{A. Vortices in the FM phase}

The atom-atom interaction in the spin-1 BEC is in the FM regime if the sign of the spin-dependent contribution is negative, such that $c_{2}<0$ in Eq. (1). Then the interaction strives to maximize the magnitude of the spin: $|\langle\hat{\mathbf{F}}\rangle|=1$ everywhere when the spin texture is uniform. We can write such a state simply as $\zeta^{(1)}=(-1,0,0)^{T}$, such that the spin vector is parallel to the $z$ axis. (The physically insignificant overall minus sign in $\zeta^{(1)}$ is included for later convenience.) Any other FM spinor can then be reached by a spin rotation, defined by three Euler angles $\alpha, \beta$, and $\gamma$, and a condensate phase $\tau$, yielding

$$
\zeta^{\mathrm{f}}=\frac{-e^{i(\tau-\gamma)}}{\sqrt{2}}\left(\begin{array}{c}
\sqrt{2} e^{-i \alpha} \cos ^{2} \frac{\beta}{2} \\
\sin \beta \\
\sqrt{2} e^{i \alpha} \sin ^{2} \frac{\beta}{2}
\end{array}\right) .
$$

The third Euler angle and the condensate phase can be combined into $\tau^{\prime}=\tau-\gamma$. The FM order-parameter manifold is then the group of rotations in three dimensions, $\mathrm{SO}(3)$, and the Euler angles $\alpha$ and $\beta$ give the local expectation value of the spin vector

$$
\langle\hat{\mathbf{F}}\rangle=\cos \alpha \sin \beta \hat{\mathbf{x}}+\sin \alpha \sin \beta \hat{\mathbf{y}}+\cos \beta \hat{\mathbf{z}} .
$$

We can classify vortices by considering how the order parameter changes on a loop around the vortex line [62]. For the FM order-parameter space $\mathrm{SO}(3)$ this gives two types of vortices: nonsingular and singular, singly quantized vortices. As a consequence of the expanded broken symmetry of the spinor wave function, the mass circulation alone is not necessarily quantized in the FM phase. Since the superfluid velocity in the FM phase is expressed in terms of $\alpha, \beta$, and $\tau^{\prime}$ as

$$
\mathbf{v}=\frac{\hbar}{m}\left(\nabla \tau^{\prime}-\cos \beta \nabla \alpha\right),
$$

a continuous variation in $\beta$ enables the circulation, $v$, to take a value anywhere in the continuous interval $\oint \nabla\left(\tau^{\prime}-\alpha\right) \cdot d \mathbf{r} \leqslant$ $v \leqslant \oint \nabla\left(\tau^{\prime}+\alpha\right) \cdot d \mathbf{r}$. In the lowest-energy configurations of both the singular and the nonsingular FM vortices, $\beta$ varies spatially [26], such that the circulation is, in general, not quantized (except when a magnetization $M= \pm 1$ forces the condensate to the one-component limit).

A nontrivial, nonsingular vortex-a coreless vortex-may be constructed by combining a $2 \pi$ rotation of the spin vector with a simultaneous $2 \pi$ winding of the condensate phase, corresponding to the choice $\alpha=\tau^{\prime}=\varphi$, where $\varphi$ is the 
azimuthal angle, in Eq. (11):

$$
\zeta^{\mathrm{cl}}=\frac{-1}{\sqrt{2}}\left(\begin{array}{c}
\sqrt{2} \cos ^{2} \frac{\beta(\rho)}{2} \\
e^{i \varphi} \sin \beta(\rho) \\
\sqrt{2} e^{2 i \varphi} \sin ^{2} \frac{\beta(\rho)}{2}
\end{array}\right) .
$$

Here $\beta$ describes how the spin vector tilts away from the $z$ axis as the radial distance $\rho$ increases. This results in a characteristic fountainlike spin texture,

$$
\langle\hat{\mathbf{F}}\rangle=\sin \beta \hat{\boldsymbol{\rho}}+\cos \beta \hat{\mathbf{z}},
$$

that is continuous everywhere, with $\langle\hat{\mathbf{F}}\rangle=\hat{\mathbf{z}}$ on the vortex line.

The coreless vortex is similar to the Anderson-ToulouseChechetkin and Mermin-Ho vortices in superfluid ${ }^{3} \mathrm{He}$ [6365], where a circulation-carrying nonsingular texture is formed by the angular-momentum vector $\mathbf{l}$ of the Cooper pairs. The two vortices have different asymptotic l-texture away from the vortex line: antiparallel to the vortex line in the AndersonToulouse-Chechetkin texture and perpendicular to it in the Mermin-Ho texture.

Owing to the effectively two-dimensional (2D) structure of the coreless spin texture, it is possible to define a winding number

$$
W=\frac{1}{8 \pi} \int_{\mathcal{S}} d \Omega_{i} \epsilon_{i j k} \hat{\mathbf{n}}_{F} \cdot\left(\frac{\partial \hat{\mathbf{n}}_{F}}{\partial x_{j}} \times \frac{\partial \hat{\mathbf{n}}_{F}}{\partial x_{k}}\right) .
$$

Here the integral is evaluated over a surface $\mathcal{S}$ that covers the full cross section of the coreless-vortex texture. We have defined $\hat{\mathbf{n}}_{F}=\langle\hat{\mathbf{F}}\rangle /|\langle\hat{\mathbf{F}}\rangle|$ as a unit vector in the direction of the local spin vector in order to later consider coreless textures where the condensate is not everywhere strictly in the FM phase. The charge $W$ defines a topological invariant if the boundary condition on $\hat{\mathbf{n}}_{F}$ away from the vortex is fixed, e.g., by physical interaction or energetics. When the asymptotic texture is uniform, $W$ is an integer (representing a mapping of the spin texture on a compactified $2 \mathrm{D}$ plane onto the unit sphere). If no boundary condition is imposed, the texture can unwind to the vortex-free state by purely local transformations of the wave function. As a result of (16), coreless textures are also called 2D "baby Skyrmions" [66] in analogy with the 3D Skyrmions [67], which represent stable particlelike solitons that can also exist in atomic BECs $[42,44,45,47,51,52]$.

The spin-1 coreless vortex may be stabilized by rotation as the bending angle $\beta(\rho)$ in Eq. (14), and therefore the superfluid circulation, adapts to minimize the energy. The asymptotic behavior of the spin texture is then determined by the imposed rotation. The spin texture away from the vortex line may also be determined by interactions with other vortices, e.g., in the formation of a composite defect. By finding $\langle\hat{\mathbf{F}}\rangle$ from Eqs. (14) and (15), and substituting in Eq. (16), we may evaluate $W$ assuming cylindrical symmetry. Taking $R$ to be the radial extent of the spin texture, we find

$$
W=\frac{1-\cos \beta(R)}{2},
$$

where we have used $\beta=0$ on the $z$ axis, such that $\left.\hat{\mathbf{n}}_{F}\right|_{\rho=0}=\hat{\mathbf{z}}$. The winding number now depends on the asymptotic value of $\beta(\rho)$, such that for $\beta(R)=\pi$ (AndersonToulouse-Chechetkin-like texture) $W=1$, and for $\beta(R)=$ $\pi / 2$ (Mermin-Ho-like texture) $W=1 / 2$.
The only other class of vortices in the FM phase is formed by the singly quantized vortices. We construct a simple representative as a $2 \pi$ winding of the condensate phase in a uniform spin texture, such that $\tau^{\prime}=\varphi$ in Eq. (11), leaving $\alpha=\alpha_{0}$ and $\beta=\beta_{0}$ constant:

$$
\zeta^{\mathrm{s}}=\frac{-e^{i \varphi}}{\sqrt{2}}\left(\begin{array}{c}
\sqrt{2} e^{-i \alpha_{0}} \cos ^{2} \frac{\beta_{0}}{2} \\
\sin \beta_{0} \\
\sqrt{2} e^{i \alpha_{0}} \sin ^{2} \frac{\beta_{0}}{2}
\end{array}\right) .
$$

Vortices in the same equivalence class can be transformed into each other by local spin rotations. For example, $\langle\hat{\mathbf{F}}\rangle$ may form a radial disgyration, with $\alpha=\varphi, \tau^{\prime}=0$, and constant $\beta=\beta_{0}$, represented by

$$
\zeta^{\mathrm{sv}}=\frac{-1}{\sqrt{2}}\left(\begin{array}{c}
\sqrt{2} e^{-i \varphi} \cos ^{2} \frac{\beta_{0}}{2} \\
\sin \beta_{0} \\
\sqrt{2} e^{i \varphi} \sin ^{2} \frac{\beta_{0}}{2}
\end{array}\right) .
$$

For $\beta_{0}=\pi / 2$ the spins lie in the $(x, y)$ plane, forming a spin vortex. Local spin rotations in a singular-vortex state may occur, for example, as a result of energy relaxation of the vortex core [26].

The spatially varying spin texture of the relaxed vortex state determines the longitudinal magnetization of the condensate. It is therefore a nontrivial question how conservation of a given initial magnetization in an experimentally prepared vortex state affects the spin texture and vortex core structure of relaxed state.

\section{B. Polar phase}

The polar phase minimizes the magnitude of the spin, $|\langle\hat{\mathbf{F}}\rangle|=0$ everywhere in a uniform spin texture, and is energetically preferred when $c_{2}>0$ in Eq. (1). Similarly to the description of the FM phase, we find an expression for a general polar state by applying a spin rotation and a condensate phase to a representative polar spinor, which we take to be $\zeta^{(0)}=(-1 / \sqrt{2}, 0,1 / \sqrt{2})^{T}$. The reason for this particular choice becomes clear in Sec. V. In terms of the condensate phase and the three Euler angles defining the spin rotation, the general polar spinor is then

$$
\zeta^{\mathrm{p}}=\frac{e^{i \tau}}{\sqrt{2}}\left(\begin{array}{c}
e^{-i \alpha}\left(e^{i \gamma} \sin ^{2} \frac{\beta}{2}-e^{-i \gamma} \cos ^{2} \frac{\beta}{2}\right) \\
-\sqrt{2} \sin \beta \cos \gamma \\
e^{i \alpha}\left(e^{i \gamma} \cos ^{2} \frac{\beta}{2}-e^{-i \gamma} \sin ^{2} \frac{\beta}{2}\right)
\end{array}\right) .
$$

Note that $e^{-i \tau} \zeta_{+}=-e^{i \tau} \zeta_{-}^{*}$. Equation (20) can therefore be written in the form [36]

$$
\zeta^{\mathrm{p}}=\frac{e^{i \tau}}{\sqrt{2}}\left(\begin{array}{c}
-d_{x}+i d_{y} \\
\sqrt{2} d_{z} \\
d_{x}+i d_{y}
\end{array}\right)
$$

where $\hat{\mathbf{d}}=d_{x} \hat{\mathbf{x}}+d_{y} \hat{\mathbf{y}}+d_{z} \hat{\mathbf{z}}$ is a unit vector. In our choice of representative spinor $\zeta^{(0)}, \hat{\mathbf{d}}=\hat{\mathbf{x}}$, and it follows that in the general polar spinor, $\hat{\mathbf{d}}=(\cos \alpha \cos \beta \cos \gamma-\sin \alpha \sin \gamma) \hat{\mathbf{x}}+$ $(\sin \alpha \cos \beta \cos \gamma+\cos \alpha \sin \gamma) \hat{\mathbf{y}}-\cos \gamma \sin \beta \hat{\mathbf{z}}$ in terms of the Euler angles. Two angles (polar and azimuthal) are sufficient to give the direction of a vector. However, anticipating the construction of phase-mixing vortex states in Sec. V, we 
choose to retain the three Euler angles, keeping in mind that more than one combination may yield the same $\hat{\mathbf{d}}$. Note that $\zeta^{\mathrm{p}}(\tau, \hat{\mathbf{d}})=\zeta^{\mathrm{p}}(\tau+\pi,-\hat{\mathbf{d}})$. This property is called nematic order [18], and the vector $\hat{\mathbf{d}}$, called the nematic axis, is understood as unoriented.

From Eq. (20) one can show that the superfluid velocity in the polar phase is given by

$$
\mathbf{v}=\frac{\hbar}{m} \nabla \tau \text {. }
$$

It then follows by the standard argument (see, e.g., Ref. [13]) that circulation must be quantized. As in the FM phase, a singly quantized vortex can be constructed as a $2 \pi$ winding of the condensate phase: $\tau=\varphi$ in Eq. (20) and keeping $\alpha=\alpha_{0}$, $\beta=\beta_{0}$, and $\gamma=\gamma_{0}$ constant to form

$$
\zeta^{1 \mathrm{q}}=\frac{e^{i \varphi}}{\sqrt{2}}\left(\begin{array}{c}
e^{-i \alpha_{0}}\left(e^{i \gamma_{0}} \sin ^{2} \frac{\beta_{0}}{2}-e^{-i \gamma_{0}} \cos ^{2} \frac{\beta_{0}}{2}\right) \\
-\sqrt{2} \sin \beta_{0} \cos \gamma_{0} \\
e^{i \alpha_{0}}\left(e^{i \gamma_{0}} \cos ^{2} \frac{\beta_{0}}{2}-e^{-i \gamma_{0}} \sin ^{2} \frac{\beta_{0}}{2}\right)
\end{array}\right) .
$$

However, because the superfluid velocity depends only on the condensate phase $\tau$, a singly quantized vortex may also include, e.g., a $2 \pi$ winding of $\alpha$ without changing the circulation. The vortex is then represented by

$$
\zeta^{1 \mathrm{q}^{\prime}}=\frac{1}{\sqrt{2}}\left(\begin{array}{c}
\left(e^{i \gamma_{0}} \sin ^{2} \frac{\beta_{0}}{2}-e^{-i \gamma_{0}} \cos ^{2} \frac{\beta_{0}}{2}\right) \\
-\sqrt{2} e^{i \varphi} \sin \beta_{0} \cos \gamma_{0} \\
e^{i 2 \varphi}\left(e^{i \gamma_{0}} \cos ^{2} \frac{\beta_{0}}{2}-e^{-i \gamma_{0}} \sin ^{2} \frac{\beta_{0}}{2}\right)
\end{array}\right)
$$

keeping $\beta=\beta_{0}$ and $\gamma=\gamma_{0}$ constant.

Unlike a scalar BEC, the singly quantized vortex in the polar spin-1 BEC does not represent the smallest unit of circulation. The nematic order makes it possible to form a vortex carrying half a quantum of circulation by allowing the condensate phase to wind by only $\pi$ as the vortex line is encircled. A simultaneous spin rotation of $\hat{\mathbf{d}}$ into $-\hat{\mathbf{d}}$ keeps the order parameter single valued. If the vortex line is on the $z$ axis, and $\hat{\mathbf{d}}$ is in the $(x, y)$ plane, this corresponds to choosing $\tau=\alpha=\varphi / 2$ and $\beta=\pi / 2$ in Eq. (20), such that

$$
\zeta^{\mathrm{hq}}=\frac{e^{i \varphi / 2}}{\sqrt{2}}\left(\begin{array}{c}
-e^{-i \varphi / 2} \\
0 \\
e^{i \varphi / 2}
\end{array}\right)=\frac{1}{\sqrt{2}}\left(\begin{array}{c}
-1 \\
0 \\
e^{i \varphi}
\end{array}\right) .
$$

Similar half-quantum vortices arise from the nematic order in superfluid ${ }^{3} \mathrm{He}-A[68,69]$ and are also found in nematic liquid crystals [70].

The quantization of circulation in the polar phase prevents angular-momentum carrying nonsingular vortices from forming. Nevertheless, it is possible for the $\hat{\mathbf{d}}$ vector to form a nonsingular texture similar to the spin texture of the coreless vortex. It does not, however, carry mass circulation. Such a nematic coreless vortex is constructed as a $2 \pi$ spin rotation, corresponding to $\alpha=\varphi$ and $\gamma=\pi$ in Eq. (20):

$$
\zeta^{\mathrm{ncv}}=\frac{1}{\sqrt{2}}\left(\begin{array}{c}
e^{-i \varphi} \cos \beta(\rho) \\
\sqrt{2} \sin \beta(\rho) \\
-e^{i \varphi} \cos \beta(\rho)
\end{array}\right) .
$$

As in the FM coreless vortex, $\beta(\rho)$ characterizes the bending of $\hat{\mathbf{d}}$ away from the $z$ axis. To form the fountainlike texture, we require $\beta(0)=\pi / 2$ and increasing monotonically. The winding number $W$ associated with the nematic texture may be defined by taking $\hat{\mathbf{n}}_{F}=\hat{\mathbf{d}}$ in Eq. (16). Note that due to the equivalence $\hat{\mathbf{d}} \leftrightarrow-\hat{\mathbf{d}}$ the sign of $W$ is no longer well defined. For the cylindrically symmetric fountain texture (43), the integral in Eq. (16) can be evaluated to yield

$$
W=\frac{1-\cos \beta^{\prime}(R)}{2}=\frac{1-\sin \beta(R)}{2},
$$

where $\beta^{\prime}=\beta-\pi / 2$ is the angle between $\hat{\mathbf{d}}$ and the $z$ axis, such that $\beta^{\prime}=0$ on the symmetry axis, where $\hat{\mathbf{d}}=\hat{\mathbf{z}}$. The value of $\hat{\mathbf{d}}$ is not fixed at the boundary, and the coreless nematic texture may smoothly dissolve. Unlike the spin texture of the FM coreless vortex, it cannot be stabilized by rotation, due to its vanishing mass circulation.

\section{Preparation of vortex states}

Several methods for controlled creation of vortex states by transferring angular momentum from an electromagnetic field have been proposed theoretically [71-76]. Some of these methods have also been implemented in experiment [77-81]. Specifically in spinor BECs, switching of a magnetic field along the symmetry axis of the trap has been used to create coreless [6,9] and nematic coreless [8,9] vortices. Two different methods have been demonstrated for controlled preparation of nonsingular vortices. Here we give a brief overview of each.

In Refs. [6,9] a coreless vortex was prepared using a time-dependent magnetic field to induce spin rotations. This technique was first proposed theoretically in Refs. [74,75] and was also implemented experimentally to prepare singly and doubly quantized vortices in a spin-polarized BEC [78,79].

The creation of a coreless vortex in the spin-1 BEC begins with a condensate prepared in a fully spin-polarized state, which we take to be $\zeta^{(1)}=(-1,0,0)^{T}$ [82]. The condensate is subject to an external 3D magnetic quadrupole field [9],

$$
\mathbf{B}=B^{\prime} \rho \hat{\boldsymbol{\rho}}+\left[B_{z}(t)-2 B^{\prime} z\right] \hat{\mathbf{z}},
$$

where we have introduced cylindrical coordinates $(\rho, \varphi, z)$. The zero-field point $z=B_{z} / 2 B^{\prime}(\rho=0)$ of the quadrupole field is initially at large $z$ so that $\mathbf{B} \| \hat{\mathbf{z}}$ in the condensate.

The coreless-vortex structure is created by linearly sweeping $B_{z}(t)$ so that the zero-field point passes through the condensate. The changing $B_{z}$ causes the magnetic field away from the $z$ axis to rotate around $\hat{\varphi}$ from the $\hat{\mathbf{z}}$ to the $-\hat{\mathbf{z}}$ direction. The rate of change of the magnetic field decreases with the distance $\rho$ from the symmetry axis. Where the rate of change is sufficiently slow, the atomic spins adiabatically follow the magnetic field, corresponding to a complete transfer from $\zeta_{+}$ to $\zeta_{-}$in the laboratory frame. However, where the rate of change of the magnetic field is rapid, atomic spin rotation is no longer adiabatic. In the laboratory frame, the spins thus rotate through an angle $\beta(\rho)$, given by the local adiabaticity of the magnetic-field sweep, which increases monotonically from zero on the symmetry axis. Linearly ramping $B_{z}(t)$ thus directly implements the spin rotation

$$
\zeta^{\mathrm{i}}=e^{-i \hat{\mathbf{F}} \cdot \beta(\rho) \hat{\varphi}} \zeta^{(1)}=\zeta^{\mathrm{cl}},
$$

where $\zeta^{\mathrm{cl}}$ is given by Eq. (14), yielding the fountainlike spin texture (15) that defines the coreless vortex in the spinor BEC. In Ref. [8] the same magnetic-field rotation technique was 
applied to a condensate initially in the polar state $\zeta=(0,1,0)^{T}$ to create the nematic coreless vortex, Eq. (26).

The first controlled preparation of a nonsingular vortex [6] used a 2D quadrupole field together with an axial bias field. The magnetic field in the trap is then $\mathbf{B}(\rho, \varphi, \theta)=B_{z}(t) \hat{\mathbf{z}}+$ $B^{\prime} \rho[\cos (2 \varphi) \hat{\boldsymbol{\rho}}-\sin (2 \varphi) \hat{\boldsymbol{\varphi}}]$. By the mechanism described above, ramping of $B_{z}(t)$ then causes a spin rotation $\zeta(\mathbf{r})=$ $\exp [-i \hat{\mathbf{F}} \cdot \beta(\rho) \hat{\mathbf{n}}] \zeta^{(1)}$ about an axis $\hat{\mathbf{n}}(\varphi)=\sin \varphi \hat{\mathbf{x}}+\cos \varphi \hat{\mathbf{y}}$. The rotation yields a nonsingular spin texture exhibiting a cross disgyration, instead of the fountainlike structure. The two are topologically equivalent.

Another technique for phase imprinting a coreless vortex was recently demonstrated in Ref. [7]. In this experiment, the coreless vortex was created in the $|m= \pm 2\rangle$ and $|m=0\rangle$ magnetic sublevels of the spin-2 manifold of ${ }^{87} \mathrm{Rb}$. The phase imprinting starts with a spin-polarized condensate in the $|m=+2\rangle$ level, with a magnetic field along the $z$ axis. Collinear $\sigma^{-}$and $\sigma^{+}$polarized laser beams along the symmetry axis then couple $|m=2\rangle$ to the $|m=0\rangle$ and $|m=-2\rangle$ levels. The laser beams have Laguerre-Gaussian and Gaussian intensity profiles, respectively, so that the population transferred to the $|m=0\rangle(|m=-2\rangle)$ level picks up a $2 \pi(4 \pi)$ phase winding. The intensity minimum of the Laguerre-Gaussian beam leaves a remaining population in $|m=2\rangle$ with no phase winding. The resulting five-component spinor represents a coreless vortex with the spin structure (15) when the three nonempty levels of the five-component spinor are regarded as a (pseudo)spin- 1 system. The bending angle $\beta$ is determined by the density profiles of the nonempty spinor components. The laser beams inducing the Raman coupling of the magnetic sublevels can be tailored with a high degree of control, and the vortex structure can therefore be precisely engineered.

By accurately creating specific spin textures, phase imprinting of coreless vortices gives control over the longitudinal magnetization of the cloud, regardless of whether interactions are polar or FM. In the spin-2 coreless-vortex experiment [7], the resulting magnetization in the spin-2 manifold is measured at $M=0.64$ for an imprinted Anderson-ToulouseChechetkin-like spin texture and at $M=0.72$ for a MerminHo-like texture. In the magnetic-field rotation experiment [6] the local magnetization $\mathcal{M}(\mathbf{r})=\left[n_{+}(\mathbf{r})-n_{-}(\mathbf{r})\right] / n(\mathbf{r})$ is reported to be $\sim 0.7$ at the center of the cloud and $\sim-0.5$ at the edge. Because of the lower density in the negatively magnetized region, also this vortex can be estimated to carry a positive, nonzero magnetization $M$.

Note that the spinor vortices presented above are composed of vortex lines in the individual components of the spinor order parameter. By phase imprinting these vortex lines using existing techniques $[77,78,80]$ it would be possible to prepare also singular-vortex states.

\section{VORTEX-CORE WAVE FUNCTIONS}

The two phases of the spin-1 BEC have different orderparameter symmetries that support different topological defects. For an overview, see, e.g., Refs. [13,61]. Here we are interested in vortex states that mix the two phases. Such solutions representing coreless and nematic-coreless vortex textures appearing in the cores of singly quantized vortices were presented in Ref. [34]. Here, we expand the discussion and provide the full derivation and additional examples of phase-mixing vortex wave functions. For that purpose we write the spinor wave function as

$$
\zeta=\frac{e^{i \tau}}{2}\left(\begin{array}{c}
\sqrt{2} e^{-i \alpha}\left(e^{i \gamma} D_{-} \sin ^{2} \frac{\beta}{2}-e^{-i \gamma} D_{+} \cos ^{2} \frac{\beta}{2}\right) \\
-\left(e^{i \gamma} D_{-}+e^{-i \gamma} D_{+}\right) \sin \beta \\
\sqrt{2} e^{i \alpha}\left(e^{i \gamma} D_{-} \cos ^{2} \frac{\beta}{2}-e^{-i \gamma} D_{+} \sin ^{2} \frac{\beta}{2}\right)
\end{array}\right),
$$

where $D_{ \pm}=\sqrt{1 \pm F}$ is given in terms of the spin magnitude $|\langle\hat{\mathbf{F}}\rangle|=F$. Here $\tau, \alpha, \beta, \gamma$ are (in general spatially varying) parameters that can be used to determine the specific vortex states that we want to analyze. In the vortex states that smoothly interpolate between the FM and polar phases also $F$ will vary in space. The state (30) can also be specified by the condensate phase, the spin magnitude $F$ and an orthonormal triad with one vector in the direction of the spin. One of the remaining vectors in the triad forms the nematic axis $\hat{\mathbf{d}}$. (In the polar limit, $\hat{\mathbf{d}}$ fully specifies the state together with the condensate phase [36].)

In order to understand the origin of Eq. (30), one should consider stationary, uniform, vortex-free spinor wave functions in the presence of Zeeman energy shifts [53,83,84]. For instance, for the uniform spin profile $\langle\hat{\mathbf{F}}\rangle=F \hat{\mathbf{z}}$ we take

$$
\zeta^{F}=\frac{1}{\sqrt{2}}\left(\begin{array}{c}
-\sqrt{1+F} \\
0 \\
\sqrt{1-F}
\end{array}\right) .
$$

In this case $\hat{\mathbf{d}}=\hat{\mathbf{x}}$. The limits $F=0$ and $F=1$ yield the polar and FM phases, $\left.\zeta^{F}\right|_{F=0}=(-1 / \sqrt{2}, 0,1 / \sqrt{2})^{T}$ and $\left.\zeta^{F}\right|_{F=1}=(-1,0,0)^{T}$, respectively. The general spinor with $|\langle\hat{\mathbf{F}}\rangle|=F$ can then be reached by applying a condensate phase $\tau$ and a $3 \mathrm{D}$ spin rotation $U(\alpha, \beta, \gamma)=$ $\exp \left(-i \hat{\mathbf{F}}_{z} \alpha\right) \exp \left(-i \hat{\mathbf{F}}_{y} \beta\right) \exp \left(-i \hat{\mathbf{F}}_{z} \gamma\right)$, defined by three Euler angles, resulting in the spatially uniform version of Eq. (30).

\section{A. Vortex core filling}

The FM and polar phases of the spin-1 BEC mix when the energy of singular vortices relaxes. As discussed in Sec. II, when the spin healing length exceeds the density healing length, it is energetically favorable for the vortex core to fill with atoms in the opposite phase. We can now explicitly construct vortex wave functions that represent the full, continuous interpolation between the vortex and its filled, nonrotating core.

\section{Polar vortices}

Consider a singly quantized vortex in a polar condensate. When the core fills, the wave function must reach the FM phase on the singularity of the polar order parameter. Consider the choice $\tau=\gamma=\varphi$ and $\alpha=0$, with constant $\beta=\beta_{0}$, in Eq. (30). We then obtain

$$
\zeta=\frac{e^{i \varphi}}{2}\left(\begin{array}{c}
\sqrt{2}\left(e^{i \varphi} \sin ^{2} \frac{\beta_{0}}{2} D_{-}-e^{-i \varphi} \cos ^{2} \frac{\beta_{0}}{2} D_{+}\right) \\
\sin \beta_{0}\left(e^{i \varphi} D_{-}+e^{-i \varphi} D_{+}\right) \\
\sqrt{2}\left(e^{i \varphi} \cos ^{2} \frac{\beta_{0}}{2} D_{-}-e^{-i \varphi} \sin ^{2} \frac{\beta_{0}}{2} D_{+}\right)
\end{array}\right),
$$

which reduces to Eq. (24), with $\gamma_{0}=0$, in the $F=0$ limit, representing a singly quantized polar vortex with a $2 \pi$ winding in $\hat{\mathbf{d}}$. In the limit $F=1$, on the other hand, Eq. (32) represents 
the vortex-free FM phase. By allowing $F(\rho)$ to decrease monotonically from $F(\rho \rightarrow 0)=1$ to $F=0$ away from the vortex line, we find the spinor representing a polar vortex with a FM core.

We can confirm this interpretation by studying the superfluid circulation. A general expression for the superfluid velocity may be derived from Eq. (30) as

$$
\mathbf{v}=\frac{\hbar}{m \rho}(\nabla \tau-F \nabla \gamma-F \cos \beta \nabla \alpha) .
$$

Since we here assume axial symmetry, we consider the mass circulation on a path at constant $\rho$,

$$
v=\oint d \mathbf{r} \cdot \mathbf{v}=\frac{h}{2 m}(l-q F-p F \cos \beta),
$$

where in our construction $l=2 d \tau / d \varphi, p=2 d \gamma / d \varphi$, and $q=2 d \alpha / d \varphi$ are integers. For the vortex (32), we then obtain $v=h(1-F) / m$, and we see that this interpolates smoothly between the noncirculating FM core $(F=1)$ and the single quantum of circulation in the polar phase $(F=0)$. Both numerical simulations [26] and experimental observations [5] have demonstrated how the axisymmetry of this singly quantized vortex breaks under dissipation and leads to the formation of a pair of half-quantum vortices.

The half-quantum vortex, described by Eq. (25), is likewise singular, and its core may fill with the FM phase. This state may be constructed from Eq. (30) by choosing $\tau=\varphi / 2, \gamma=$ $\pi+\varphi / 2$, and $\alpha=0$ with constant $\beta=\beta_{0}$ to yield

$$
\zeta=\frac{e^{i \frac{\varphi}{2}}}{2}\left(\begin{array}{c}
\sqrt{2}\left(e^{-i \frac{\varphi}{2}} \cos ^{2} \frac{\beta_{0}}{2} D_{+}-e^{i \frac{\varphi}{2}} \sin ^{2} \frac{\beta_{0}}{2} D_{-}\right) \\
\sin \beta_{0}\left(e^{-i \frac{\varphi}{2}} D_{+}+e^{i \frac{\varphi}{2}} D_{-}\right) \\
\sqrt{2}\left(e^{-i \frac{\varphi}{2}} \sin ^{2} \frac{\beta_{0}}{2} D_{+}-e^{i \frac{\varphi}{2}} \cos ^{2} \frac{\beta_{0}}{2} D_{-}\right)
\end{array}\right)
$$

The $F=1$ limit is the vortex-free FM phase with $\langle\hat{\mathbf{F}}\rangle=$ $\sin \beta_{0} \hat{\mathbf{x}}+\cos \beta_{0} \hat{\mathbf{z}}$. By allowing $F(\rho)$ to decrease monotonically with the radial distance from $F=1$ to $F=0$, the circulation, $v=h(1-F) / 2 m$ from Eq. (34), smoothly interpolates between the inner, noncirculating FM phase and the outer polar half-quantum vortex.

\section{FM vortices}

Also in the FM phase, cores of singular vortices may be filled (with atoms in the polar phase) through the same mechanism. In Eq. (19), representing a singular FM vortex, $\zeta_{0}$ is nonsingular everywhere and can therefore fill the vortex core. We now generalize this solution to a spinor that interpolates between an outer singular FM vortex and an inner polar core. We choose $\alpha=\varphi$ and $\tau=0$ with arbitrary $\gamma=\gamma_{0}$, yielding

$$
\zeta=\frac{1}{2}\left(\begin{array}{c}
\sqrt{2} e^{-i \varphi}\left(e^{i \gamma_{0}} \sin ^{2} \frac{\beta}{2} D_{-}-e^{-i \gamma_{0}} \cos ^{2} \frac{\beta}{2} D_{+}\right) \\
-\sin \beta\left(e^{i \gamma_{0}} D_{-}+e^{-i \gamma_{0}} D_{+}\right) \\
\sqrt{2} e^{i \varphi}\left(e^{i \gamma_{0}} \cos ^{2} \frac{\beta}{2} D_{-}-e^{-i \gamma_{0}} \sin ^{2} \frac{\beta}{2} D_{+}\right)
\end{array}\right) .
$$

In the $F=0$ limit, the corresponding circulation $v=$ $-h F \cos \beta / m$ vanishes, and the spinor represents a noncirculating polar condensate with $\hat{\mathbf{d}}=\cos \beta \hat{\boldsymbol{\rho}}-\sin \beta \hat{\mathbf{z}}$.

\section{B. Composite topological defects}

The vortex wave functions constructed above, simultaneously representing a singular vortex and its core, are summarized in the first, second, and sixth rows of Table I. We give the corresponding choices for the condensate phase and spin rotations, as well as the mass circulation.

We now generalize the construction of phase-mixing spinors to allow vortex states in both polar and FM limits. The wave function may then, for example, represent a defect configuration that exhibits different small- and large-distance topology of the vortex core. Such composite topological defects exhibit a hierarchy of core structures, between which the wave function interpolates smoothly with radial distance from the vortex line. We provide explicit examples with both singular and nonsingular inner cores as shown in Table I. The interpolation between the two solutions is reminiscent of the interfaces between spatially separated, coexisting phases of a superfluid system. Constructions analogous to those in Table I can then be used to describe wave functions that continuously connect defects across the topological interface [40,41].

By making appropriate choices for the Euler angles and condensate phase in Eq. (30), a large family of nontrivial wave functions connecting polar and FM vortex states may thus be constructed. In Table I we list the basic FM- and polarlimit vortex states and provide the $\tau, \alpha$, and $\gamma$ needed to construct them from Eq. (30) as half-integer (or integer, for $\alpha$ ) multiples of the azimuthal angle $\varphi$. The rightmost column gives the superfluid circulation as a function of $F$ and $\beta$. Note that in addition to the filled-core vortex states, we are now able construct inner cores with nontrivial, nonsingular textures. Such states become relevant, for example, when a FM coreless vortex is phase imprinted on a polar condensate [34]. In addition, we also find more exotic wave functions that connect singular vortices, as well as a smooth connection of nontrivial, nonsingular textures.

In Sec. VI we demonstrate numerically how, in addition to the filled cores of singular vortices (Sec. VII), a singly quantized polar vortex with coreless-vortex FM core may be energetically stable and that a FM vortex with nematic coreless vortex core may be stable in an effective two-component regime at strong magnetization. Singular vortices with singular inner cores do not naturally appear as the ground state, but we show how they may be stabilized through precise control over the quadratic Zeeman shift in Sec. VIII.

In the following we analyze in detail the construction of those composite defect states particularly relevant for the analysis of our numerical results. Some additional constructions and discussion are given in the Appendix.

\section{Singly quantized polar vortex with coreless-vortex core}

As a first example, we construct a spinor wave function representing a FM coreless vortex that appears as the core of a polar vortex, as given in Ref. [34]. This vortex structure is analyzed numerically in Sec. VI A 2. In Eq. (30) we choose $\tau=\alpha=\varphi$ and $\gamma=0$ to yield

$$
\zeta^{\mathrm{cl}}=\frac{1}{2}\left(\begin{array}{c}
\sqrt{2}\left(D_{-} \sin ^{2} \frac{\beta}{2}-D_{+} \cos ^{2} \frac{\beta}{2}\right) \\
-e^{i \varphi}\left(D_{-}+D_{+}\right) \sin \beta \\
\sqrt{2} e^{2 i \varphi}\left(D_{-} \cos ^{2} \frac{\beta}{2}-D_{+} \sin ^{2} \frac{\beta}{2}\right)
\end{array}\right) \text {. }
$$


TABLE I. Spinor wave functions for vortex states that continuously connect FM and polar phases can be constructed from Eq. (30). The table shows the state in the FM and polar limits and the choices for $\tau, \alpha$, and $\gamma$ necessary to construct them, given as half-integer or integer multiples of the azimuthal angle $\varphi$. The rightmost column shows the corresponding superfluid circulation $v$ as a function of spin magnitude $F$ and the second Euler angle $\beta$ (also indicating any assumed functional form for nonconstant $\beta$ where required). Note that vortex-free FM (polar) limits provide wave functions that simultaneously describe a singular polar (FM) vortex and its filled core, while solutions that exhibit vortex states in both limits correspond to composite defects.

\begin{tabular}{|c|c|c|c|c|c|}
\hline FM limit & Polar limit & $\tau / \varphi$ & $\alpha / \varphi$ & $\gamma / \varphi$ & $m v / h$ \\
\hline No vortex & Half-quantum vortex & $1 / 2$ & 0 & $1 / 2$ & $(1-F) / 2$ \\
\hline No vortex & Singly quantized vortex & 1 & 0 & 1 & $1-F$ \\
\hline Coreless vortex & Half-quantum vortex & $1 / 2$ & 1 & $-1 / 2$ & $(1+F) / 2-F \cos \beta$ \\
\hline Coreless vortex & Singly quantized vortex & 1 & 1 & 0 & $1-F \cos \beta$ \\
\hline Coreless vortex & Nematic coreless vortex & 0 & 1 & -1 & $F(1-\cos \beta)$ \\
\hline Singular vortex & No vortex & 0 & 1 & 0 & $-F \cos \beta_{0}$ \\
\hline Singular vortex & Nematic coreless vortex & 0 & 1 & 0 & $-F \cos \beta(\rho)$ \\
\hline Singular vortex & Half-quantum vortex & $1 / 2$ & 1 & $1 / 2$ & $(1-F) / 2-F \cos \beta$ \\
\hline Singular vortex & Half-quantum vortex & $1 / 2$ & 0 & $-1 / 2$ & $(1+F) / 2$ \\
\hline Singular vortex & Singly quantized vortex & 1 & 0 & 0 & 1 \\
\hline
\end{tabular}

The spin texture is then given by

$$
\langle\hat{\mathbf{F}}\rangle=F(\mathbf{r})[\sin \beta(\mathbf{r}) \hat{\boldsymbol{\rho}}+\cos \beta(\mathbf{r}) \hat{\mathbf{z}}],
$$

where $\beta(\mathbf{r})$ increases monotonically from zero on the symmetry axis to form the characteristic fountain texture with varying spin magnitude $F(\mathbf{r})$. In the limit $F=1$, we retrieve the coreless vortex represented by Eqs. (14) and (15). In the polar limit $F=0$, on the other hand, Eq. (37) represents a singly quantized vortex,

$$
\left.\zeta^{\mathrm{cl}}\right|_{F \rightarrow 0}=\frac{e^{i \varphi}}{\sqrt{2}}\left(\begin{array}{c}
-e^{-i \varphi} \cos \beta \\
-\sqrt{2} \sin \beta \\
e^{i \varphi} \cos \beta
\end{array}\right),
$$

where we have explicitly separated out the condensate phase $\tau=\varphi$. The nematic axis forms the texture $\hat{\mathbf{d}}=\cos \beta \hat{\boldsymbol{\rho}}-$ $\sin \beta \hat{\mathbf{z}}$. In general, the spin rotation that accompanies the winding of the condensate phase therefore represents a disgyration of the nematic axis.

The vortex (37) can represent a solution for which $F$ is nonuniform, so that Eqs. (14) and (39) are the two limiting solutions. We can form a composite topological defect by setting $F(\rho=0)=1$ and $\beta(\rho=0)=0$ at the center and letting $F \rightarrow 0$ and $\beta \rightarrow \pi / 2$ as $\rho$ increases. Then the core exhibits a coreless-vortex fountain texture that continuously transforms toward a singular polar vortex as the radius increases.

The mixing of the polar and FM phases in the vortex configuration is also reflected in the superfluid velocity given by Eq. (33). For the vortex state (37), this reduces to

$$
\mathbf{v}^{\mathrm{cl}}=\frac{\hbar}{m \rho}[1-F(\rho) \cos \beta(\rho)] \hat{\boldsymbol{\varphi}},
$$

when $F$ and $\beta$ depend only on the radial distance $\rho$. By considering a circular loop $\mathcal{C}$ at constant $\rho$ enclosing the vortex line, we can then compute the circulation

$$
v=\int_{\mathcal{C}} d \mathbf{r} \cdot \mathbf{v}^{\mathrm{cl}}=\frac{h}{m}[1-F(\rho) \cos \beta(\rho)] .
$$

Note that for nonzero $F$, circulation increases with increasing $\beta(\rho)$, implying that the coreless-vortex texture can adapt to an imposed rotation. This indicates that the spin texture will bend more sharply at faster rotation to provide increased angular momentum. We may regard the integrand of Eq. (41) as a circulation density,

$$
\mathcal{V}(\mathbf{r})=\mathbf{v}(\mathbf{r}) \cdot \hat{\boldsymbol{\varphi}} \rho,
$$

along a cylindrically symmetric path. The circulation of Eq. (37) continuously interpolates between the polar and FM phases, smoothly connecting the small-distance and largedistance topology of the vortex. Note that it further follows from Eq. (41) that circulation alone is quantized only in the limit $F \rightarrow 0$.

Note that the winding number $W$, given by Eq. (16), remains defined also for the spin texture of the composite defect, provided that the surface $\mathcal{S}$ covers the full cross section of the nonpolar core. By substituting $\langle\hat{\mathbf{F}}\rangle$ from Eq. (38) into Eq. (16), we may evaluate $W$. Assuming cylindrical symmetry and taking $R$ to be the radial extent of the spin texture, $W$ is again given by Eq. (17).

\section{Singular FM vortex with nematic coreless vortex core}

In Sec. VIB we numerically investigate the stability of the nematic coreless vortex constructed in Eq. (26). In order for the vortex to be stabilized by rotation, the condensate must develop nonpolar, circulation-carrying regions. We therefore generalize the nematic coreless-vortex solution to the spinor wave function given in Ref. [34] that also allows nonzero spin. We note that we wish to construct a vortex where

$$
\hat{\mathbf{d}}=\sin \beta^{\prime}(\rho) \hat{\boldsymbol{\rho}}+\cos \beta^{\prime}(\rho) \hat{\mathbf{z}},
$$

corresponding to the state phase imprinted by Choi et al. [8,9]. The angle $\beta^{\prime}$ between $\hat{\mathbf{d}}$ and the $z$ axis increases from $\beta^{\prime}=0$ at $\rho=0$ to $\beta^{\prime}=\pi / 2\left(\beta^{\prime}=\pi\right)$ at the edge for a Mermin-Ho-like (Anderson-Toulouse-Chechetkin-like) texture. Note that since the Euler angles in Eq. (30) represent spin rotations of Eq. (31), we have $\beta(\rho)=\beta^{\prime}(\rho)+\pi / 2$, such that $\beta=\pi / 2$ at the center of the vortex. The desired vortex state can then be constructed from Eq. (30) by additionally choosing $\alpha=\varphi, \gamma=\pi$, and 
$\tau=0$ to yield

$$
\zeta^{\mathrm{n}}=\frac{1}{2}\left(\begin{array}{c}
\sqrt{2} e^{-i \varphi}\left(D_{+} \cos ^{2} \frac{\beta}{2}-D_{-} \sin ^{2} \frac{\beta}{2}\right) \\
\left(D_{+}+D_{-}\right) \sin \beta \\
\sqrt{2} e^{i \varphi}\left(D_{+} \sin ^{2} \frac{\beta}{2}-D_{-} \cos ^{2} \frac{\beta}{2}\right)
\end{array}\right),
$$

with spin profile $\langle\hat{\mathbf{F}}\rangle=F[\sin \beta(\rho) \hat{\boldsymbol{\rho}}+\cos \beta(\rho) \hat{\mathbf{z}}]$. Note that this is a special case of Eq. (36), with the angle $\beta(\rho)$ chosen as a monotonically increasing function of $\rho$ to yield the desired fountain texture (43) in $\hat{\mathbf{d}}$.

In a magnetized BEC, Eq. (44) can represent a composite vortex that mixes the FM and polar phases. We consider a solution for which $F$ exhibits a spatial structure interpolating between $F \rightarrow 0$ at the center and $F \rightarrow 1$ at the edge of the cloud. In the limit $F \rightarrow 1$, Eq. (44) becomes a singular singly quantized FM vortex,

$$
\left.\zeta^{\mathrm{n}}\right|_{F \rightarrow 1}=\frac{1}{\sqrt{2}}\left(\begin{array}{c}
\sqrt{2} e^{-i \varphi} \cos ^{2} \frac{\beta}{2} \\
\sin \beta \\
\sqrt{2} e^{i \varphi} \sin ^{2} \frac{\beta}{2}
\end{array}\right) .
$$

\section{Singular FM vortex with singular polar-vortex core}

It is also possible to construct composite topological defects where both the inner and the outer vortices are singular (leaving open the question of the core structure of the inner singular vortex). We demonstrate in Sec. VIII how such structures may be stabilized using a tunable quadratic Zeeman shift. The simplest example to construct is that of a singular FM vortex [Eq. (18)], forming the core of the singly quantized polar vortex [Eq. (23)], or vice versa. (The more complicated connection of a singular FM vortex to a polar half-quantum vortex is provided in the Appendix.) We construct the corresponding spinor by setting $\tau=\varphi$ and $\alpha=0$. For any constant $\gamma=\gamma_{0}$ and $\beta=\beta_{0}$, we then have

$$
\zeta=\frac{e^{i \varphi}}{2}\left(\begin{array}{c}
\sqrt{2}\left(e^{i \gamma_{0}} \sin ^{2} \frac{\beta_{0}}{2} D_{-}-e^{-i \gamma_{0}} \cos ^{2} \frac{\beta_{0}}{2} D_{+}\right) \\
-\sin \beta_{0}\left(e^{i \gamma_{0}} D_{-}+e^{-i \gamma_{0}} D_{+}\right) \\
\sqrt{2}\left(e^{i \gamma_{0}} \cos ^{2} \frac{\beta_{0}}{2} D_{-}-e^{-i \gamma_{0}} \sin ^{2} \frac{\beta_{0}}{2} D_{+}\right)
\end{array}\right),
$$

with circulation $v=h / m$. This vortex is singular for all values of $F$ and $\beta$, such that the singularity cannot be avoided by judicious choice of parameters. In the $F=1$ limit then, Eq. (46) describes a singular FM vortex, with uniform spin texture $\langle\hat{\mathbf{F}}\rangle=\sin \beta_{0} \hat{\mathbf{x}}+\cos \beta_{0} \hat{\mathbf{z}}$. The $F=0$ limit similarly corresponds to a singular polar vortex with uniform $\hat{\mathbf{d}}=$ $\cos \beta_{0} \cos \gamma_{0} \hat{\mathbf{x}}+\sin \gamma_{0} \hat{\mathbf{y}}-\sin \beta_{0} \cos \gamma_{0} \hat{\mathbf{z}}$. A wave function that continuously interpolates between the two singular vortices is then constructed by taking $F(\rho)$ to vary monotonically between $F=0$ and $F=1$. The boundary condition on $F(\rho)$ away from the vortex line determines the large-distance topology.

\section{NONSINGULAR VORTICES AND TEXTURES}

Nonsingular vortices have been prepared in several recent experiments [6-9]. However, in Refs. [6,9] a FM coreless vortex was prepared (using the magnetic-field rotation technique described in Sec. IV C) in ${ }^{23} \mathrm{Na}$, which exhibits polar interactions, in which case the vortex would not be expected to be stable by simple energetic arguments alone. In Ref. [34], we demonstrated that conservation of magnetization can stabilize the imprinted coreless vortex, and at large magnetization also a nematic coreless vortex. We noted that it may also destabilize the coreless vortex in a FM condensate. In the following, we first expand the discussion of the coreless vortex in the FM interaction regime and determine in detail how the energetically stable structure varies as magnetization is conserved at different values. In particular, we explain how conservation of a sufficiently weak magnetization can destabilize the coreless-vortex texture, such that a singular vortex instead forms the ground state in the rotating FM condensate. We then explain how conservation of magnetization can also energetically stabilize the coreless vortex when it is phase imprinted on a BEC in the polar regime [34]. Equation (24) will then allow us to understand the stable coreless vortex as the composite core of a singly quantized vortex in the polar BEC. We also investigate whether conservation of magnetization can stabilize a nematic coreless vortex and explain our finding [34] that stability is achieved only at strong magnetization in an effective two-component regime.

\section{A. Coreless vortex}

\section{FM regime}

We first study the stability and spin texture of the coreless vortex as it relaxes under conservation of magnetization in the FM interaction regime. The initial state in our numerical energy relaxation is the coreless vortex as created in phaseimprinting experiments, Eq. (14). The initial state is in the FM phase everywhere, with $\beta(\rho)$ specifying the initial magnetization. In the FM interaction regime, the condensate remains in the FM phase with $F=1$ everywhere during relaxation when magnetization is not conserved. We find, however, that conservation of a sufficiently weak magnetization can lead to energetic instability of the coreless vortex, with a singular vortex becoming the rotating ground state. This may seem surprising, since in studies that do not conserve magnetization in energy relaxation, coreless vortices are predicted to form the ground state at sufficiently rapid rotation [29-32]; a singular vortex may then also be energetically (meta)stable, but always has a higher energy than the coreless vortex [26]. To understand this instability of the coreless vortex, it is instructive to first consider the stable configuration of a FM coreless vortex when magnetization is not explicitly conserved in the relaxation process.

When the magnetization is not conserved, the fountain texture of the FM coreless vortex displays a characteristic radial profile of $\beta(\rho)$. As the rotation frequency increases, the angular momentum also increases. Since increased angular momentum requires a sharper bending of $\beta(\rho)$, the result is that the magnetization of the energetically stable configuration decreases as the rotation frequency increases, as illustrated in Fig. 1(f).

To understand this, we present a qualitative description of an axisymmetric vortex at the center of the trap, described by the spinor,

$$
\zeta=e^{i a \varphi}\left(\begin{array}{c}
e^{-i b \varphi}\left|\zeta_{+}(\rho)\right| \\
\left|\zeta_{0}(\rho)\right| \\
e^{i b \varphi}\left|\zeta_{-}(\rho)\right|
\end{array}\right)
$$



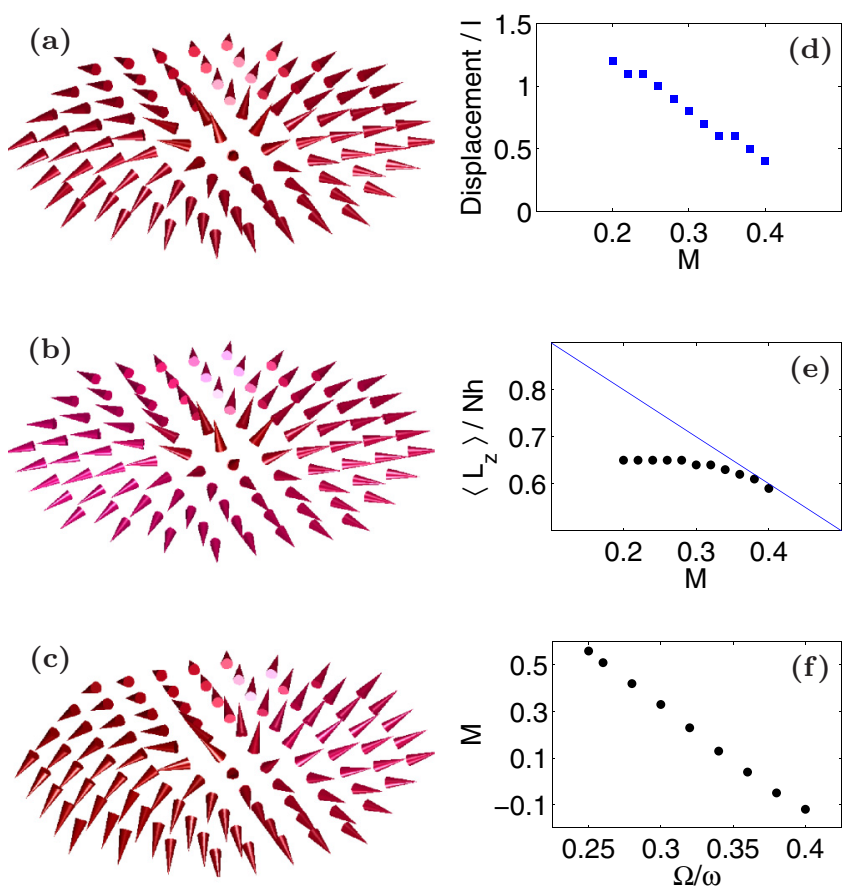

FIG. 1. Numerically calculated spin textures in the $x-y$ plane for the coreless vortex in the FM regime with an initial magnetization $M=0.4$ (a) not conserved and (b) conserved throughout the relaxation process. (c) The same for a conserved magnetization of $M=0.2$, showing a displacement of the coreless vortex relative to the more strongly magnetized case. (d) Displacement and (e) angular momentum of the coreless vortex in a trap rotating at $\Omega / \omega=0.3$ for different values of the conserved magnetization (black dots) compared with the angular momentum of an axisymmetric coreless vortex at the center of the trap, for the same magnetization (blue line). (f) Numerically calculated magnetization of the energy-minimizing coreless vortex in the FM regime as a function of rotation frequency, where magnetization-conserving relaxation has not been enforced.

where $\left|\zeta_{i}(\rho)\right|^{2}$ are the populations of the three spinor components as a function of radius, giving rise to the radial profile of $\beta$. The integers $a$ and $b$ represent the winding of the condensate phase and spin vector, respectively. The expectation value of the angular momentum for such a vortex is [85]

$$
\left\langle\hat{L}_{z}\right\rangle=(a-b) N_{+}+a N_{0}+(a+b) N_{-},
$$

which may be simplified via Eq. (3) to

$$
\left\langle\hat{L}_{z}\right\rangle=(a-b M) N .
$$

In the case of the coreless vortex, $a=b=1$ and so the angular momentum increases linearly with decreasing magnetization. This illustrates why increasing the rotation frequency decreases the magnetization when the magnetization is not conserved: Increased angular momentum acts to decrease the longitudinal magnetization in the system. This qualitative description is also useful in understanding the behavior of the coreless vortex subject to magnetization-conserving relaxation.

The fountain texture of the coreless vortex energetically favors nonzero magnetization. It is therefore instructive to first consider a conserved magnetization close to that which would

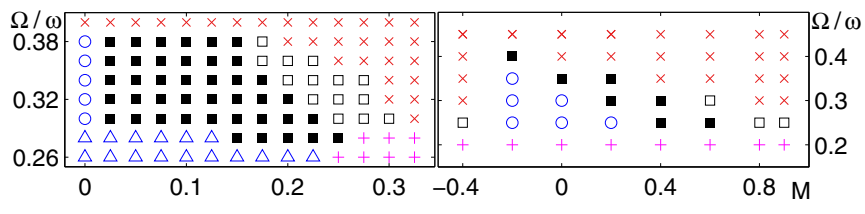

FIG. 2. Energetic stability of the coreless vortex in the polar (left) and FM (right) interaction regimes; ( $\mathbf{\square})$ stable coreless vortex; $(\square)$ stable effective two-component coreless vortex; $(\triangle)$ instability towards a half-quantum vortex; (o) pair of half-quantum vortices (polar regime) or singular vortex (FM regime); (+) vortex-free state; $(\times)$ nucleation of additional vortices.

arise if magnetization were unconstrained. At a trap-rotation frequency just above that at which the coreless vortex becomes stable, $M \sim 0.5$. Strictly conserving $M=0.5$ throughout energy relaxation then has little impact on the structure of the coreless vortex, as one would intuitively expect. Keeping the rotation frequency constant, we now study the consequences as $M$ deviates from 0.5 .

Reducing the value of the conserved magnetization leads to a displacement of the coreless vortex from the trap center, as demonstrated in Fig. 1(d). This may be understood as reducing the contribution to the magnetization arising from the center of the vortex by forcing the center of the vortex to lie in a region of lower density. At the same time, the continuous bending of the spin vector ensures that an enlarged region of negative magnetization density forms at the edge of the trap farthest from the center of the vortex. The combination of these two effects results in a reduction of the total longitudinal magnetization.

Decreasing the magnetization further causes a greater displacement of the coreless vortex as illustrated in Figs. 1(a)1(d) until, at $M \sim 0.2$, the coreless vortex is unstable towards splitting into a pair of singular vortices. We note that the magnetization at which this happens decreases as the rotation frequency increases and thus infer that it is the displacement of the coreless vortex that triggers this instability. Further displacement of the vortex would produce a vortex-free state, which for a range of rotation frequencies is higher in energy than the singular vortex. The coreless vortex therefore splits into a pair of singular vortices, one of which then exits the cloud. Contrary to the findings when magnetization is not conserved, which showed that the coreless vortex is always the ground state, we find that the singular vortex is, in fact, the ground state for sufficiently weak magnetization. The range of magnetization for which this is true decreases with increasing rotation frequency of the trap. The corresponding stability diagram is shown in Fig. 2.

One additional consequence of this displacement of the coreless vortex due to a reduction of the conserved magnetization is that the angular momentum is approximately independent of the magnetization provided that the coreless vortex remains stable, shown in Fig. 1(e). From the discussion of the axisymmetric coreless vortex at the center of the trap, we saw that decreasing the magnetization served to increase the angular momentum. However, the displacement of the vortex then reduces the angular momentum relative to the axisymmetric vortex at the center of the trap, canceling out the increased angular momentum due to the reduction in magnetization. 

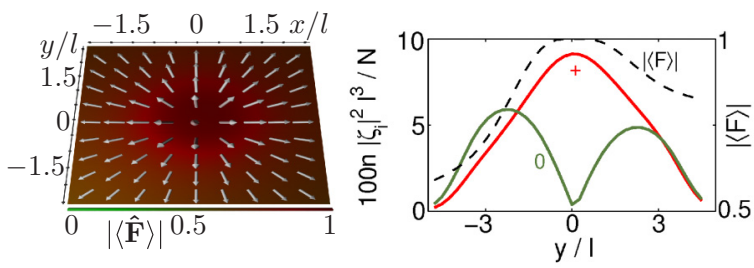

FIG. 3. Coreless vortex in strongly magnetized condensate. (Left) Spin magnitude (color scale) and spin vector (arrows). Note how the spin magnitude falls below 1 away from the vortex center. (Right) Spinor wave function along the $y$ axis, showing how the strong magnetization leads to an effective two-component regime, where $\zeta_{-}$is depleted and $\zeta_{0}$ exhibits a $2 \pi$ phase winding.

The FM coreless vortex was found to be stable for magnetization below $M \sim 0.5$. For stronger magnetization we obtain an effective two-component coreless-vortex state shown in Fig. 3, where $\zeta_{0}$ represents a singly quantized vortex whose core is filled by $\zeta_{+}$. The transition to the two-component system occurs when the $\beta(\rho)$ profile no longer allows the threecomponent vortex (14) to satisfy the magnetization constraint, resulting in depopulated $\zeta_{-}$. The threshold magnetization value decreases with rotation until at $\Omega \simeq 0.35 \omega$ the coreless vortex is stable only in the two-component regime. Despite the resulting formation of non-FM regions away from the vortex line, the state nevertheless represents a coreless vortex with a charge $W$ that may be calculated from Eq. (16).

As noted above, the FM coreless vortex in the FM regime can be unstable against splitting into a pair of singular vortices when conservation of a weak magnetization is imposed. One of these vortices then exits the cloud leaving a single, singular vortex where relaxation without explicitly conserving magnetization would predict the coreless vortex to be stable. As the magnetization becomes increasingly negative, one might naturally expect also this singular vortex to become unstable as $\zeta_{+}$is depopulated. One might further expect that the resulting vortex structure would be a singular vortex in an effective two-component regime, exhibiting a polar vortex core in an otherwise FM condensate. Perhaps surprisingly, we find that a strongly magnetized coreless vortex, similar to Fig. 3 but with negative magnetization, is stabilized in its place.

The coreless vortex first splits into the pair of singular vortices with polar cores, such that the doubly quantized vortex in $\zeta_{-}$splits into two singly quantized vortices. As the energy relaxes, $\zeta_{+}$is depopulated leaving a spinor with winding numbers 1 and 2 in $\zeta_{0}$ and $\zeta_{-}$, respectively. The vortices in $\zeta_{-}$then exit the cloud, leaving a spinor of the same form as that for strong, positive magnetization, with $\zeta_{ \pm}$interchanged. The structure is as described above with the spin rotated by $\pi$ about an arbitrary axis in the $x-y$ plane.

\section{Polar regime}

Next we study the energetic stability of a FM coreless vortex in the polar interaction regime. In spin-1 BECs of ${ }^{23} \mathrm{Na}$, which exhibit polar interactions, FM coreless vortices have been prepared experimentally via magnetic-field rotation [6,9]. Simple energetic arguments would predict that the coreless vortex is then always unstable. However, conservation of magnetization requires that the condensate always exhibit nonpolar regions, which we show allows the coreless vortex to remain stable. As an initial state we take the experimentally phase-imprinted state [Eq. (14)], with $F=1$ everywhere, for different $M$. The energetic stability and structure of the vortex is then determined by numerically minimizing the free energy in a rotating trap (at the frequency $\Omega$ ).

In a spin-1 BEC a vortex singularity can be accommodated by exciting the wave function out of its ground-state manifold, whenever it is energetically more favorable to adjust the spin value than force the density to vanish at the singular core $[26,36]$. This happens when the characteristic length scales $\xi_{n}$ and $\xi_{F}$ [Eq. (4)] satisfy $\xi_{F} \gtrsim \xi_{n}$. In addition, conservation of magnetization now introduces $\eta_{\mathrm{f}}$ [Eq. (8)] as a third length scale that describes, in an otherwise polar condensate, the size of a FM core needed to yield a specific $M$.

As the energy of the imprinted coreless vortex relaxes, the spin-dependent interaction drives the condensate towards the polar phase with $F \rightarrow 0$ away from the vortex line. The outer region then approaches the singly quantized vortex (24), exhibiting a radial disgyration of $\hat{\mathbf{d}}$. In the limit of weak magnetization, the vortex core splits into two half-quantum vortices, similarly to the splitting of a singly quantized vortex predicted when magnetization is not conserved [26] and observed in experiment [5]. At $M=0$, the fountain texture is lost entirely. When $M$ increases, the stable coreless-vortex spin texture gradually becomes more pronounced, preventing the core splitting. The vortex, shown in Fig. 4, still exhibits axial asymmetry in the magnetized core region, with two close-lying spin maxima $F=1$, and $\langle\hat{\mathbf{F}}\rangle \| \hat{\mathbf{z}}$ at the center. Ignoring the slight core asymmetry, the vortex can be qualitatively described by the analytic model (37): The spin winds to $\langle\hat{\mathbf{F}}\rangle \| \hat{\boldsymbol{\rho}}$ as $\rho$ increases. Simultaneously, $F$ decreases sharply and the configuration approaches a singly quantized polar vortex. The size of the core (the magnetization density half width at half maximum) is $\sim \eta_{\mathrm{f}}$. Comparison of length scales then suggests that the coreless texture becomes pronounced when $\eta_{\mathrm{f}} \gtrsim \xi_{F}$ $\left(>\xi_{n}\right)$, which is in qualitative agreement with our numerical results.

Owing to the trap, $F$ can reach a local minimum-which may not vanish in all directions-and start increasing at the edge of the cloud. The vortex profile then depends on $M$ and additionally, e.g., on the quadratic Zeeman shift, which favors the polar phase [84]. We may therefore envisage a scheme to engineer the core symmetry and even more complex composite defects by Laguerre-Gaussian lasers that generate a Zeeman
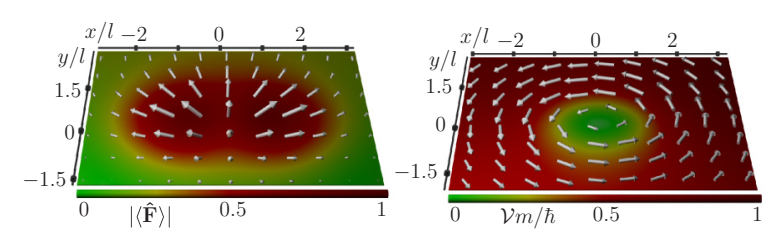

FIG. 4. (Left) Spin profile $\langle\hat{\mathbf{F}}\rangle$ (arrows) and $|\langle\hat{\mathbf{F}}\rangle|$ (color gradient and arrow lengths) of the coreless vortex in the polar regime, interpolating between FM and polar phases and displaying the characteristic fountain texture inside the core of a singular polar vortex. (Right) The corresponding superfluid velocity $\mathbf{v}$ and its magnitude (arrows) and circulation density $\mathcal{V}=\rho \mathbf{v} \cdot \hat{\boldsymbol{\varphi}}$ (color gradient) continuously interpolating from nonsingular to singular circulation. 
shift with a cylindrical shell symmetry, which we explore further in Sec. VIII.

It follows from Eq. (34) that circulation alone is quantized only in the polar phase $(F=0)$. Figure 4 demonstrates how the superfluid circulation in the relaxed vortex state smoothly connects the small- and large-distance topology, as qualitatively described by Eqs. (40)-(42) for the analytic model (37).

We note, finally, that also in the polar interaction regime the three-component coreless vortex gives way to the effective two-component state in Fig. 3 when magnetization is sufficiently strong. The transition to the two-component system occurs when the $\beta(\rho)$ profile no longer allows the three-component vortex (37) to satisfy the magnetization constraint, depopulating $\zeta_{-}$. The threshold magnetization value decreases with rotation (Fig. 2). At the lowest frequency where the coreless vortex is stable, the two-component regime appears at $M \simeq 0.25$. When the rotation is nearly rapid enough to nucleate additional vortices, the threshold magnetization has decreased to $M \simeq 0.15$. For large $c_{0}$, the stable twocomponent solution represents a smooth transition from the $F=1$ coreless vortex at the trap center to the $F=0$ singular vortex at the edge of the cloud.

\section{B. Nematic coreless vortex}

When the magnetic-field rotation technique used to phaseimprint the FM coreless vortex [6,9] is applied to a BEC prepared initially in the state $\zeta=(0,1,0)^{T}$, representing the polar phase with $\hat{\mathbf{d}}=\hat{\mathbf{z}}$ and longitudinal magnetization $M=0$, the result is a nematic coreless vortex [8,9], Eq. (26). This is characterized by a fountainlike texture $\hat{\mathbf{d}}=\sin \beta^{\prime} \hat{\boldsymbol{\rho}}+\cos \beta^{\prime} \hat{\mathbf{z}}$ of the nematic axis. Since the condensate is unmagnetized prior to the magnetic-field rotation, the longitudinal magnetization remains zero in the imprinted texture. This $\hat{\mathbf{d}}$ texture can continuously unwind to the uniform state and has vanishing mass circulation. It can therefore not be stabilized by rotation as the coreless vortex can.

We ask instead whether the nematic coreless vortex can be stable inside the core of a composite topological defect when a conserved, nonzero magnetization necessitates the formation of nonpolar regions. A nematic coreless vortex with a nonzero magnetization could be created by phase imprinting via population transfer $[7,77,80]$ that individually prepares the appropriate phase windings of $\langle-2 \pi, 0,2 \pi\rangle$ in the spinor components. In a magnetized BEC, $F$ will acquire a spatial structure interpolating between $F=0$ at the center of the cloud to $F>0$ at the edge as energy relaxes. This behavior is described in the analytic model in Eq. (44). From $\hat{\mathbf{d}} \perp\langle\hat{\mathbf{F}}\rangle$, it follows that in order to have the fountain texture in $\hat{\mathbf{d}}$ we must have $\beta=\pi / 2$ at $\rho=0$ and increasing monotonically. The corresponding mass circulation, $\oint d \mathbf{r} \cdot \mathbf{v}=-\frac{h F}{m} \cos \beta$, interpolates from the noncirculating polar core to a nonzero circulation, allowing, in principle, stabilization by rotation.

For sufficiently strong magnetization the condensate will reach $F=1$ in an outer FM region. This outer FM region represents the large-distance topology of a singular FM vortex, while the topology at small distances is represented by the nematic coreless vortex. We find that the numerical relaxation process of the phase-imprinted $\langle-2 \pi, 0,2 \pi\rangle$ vortex
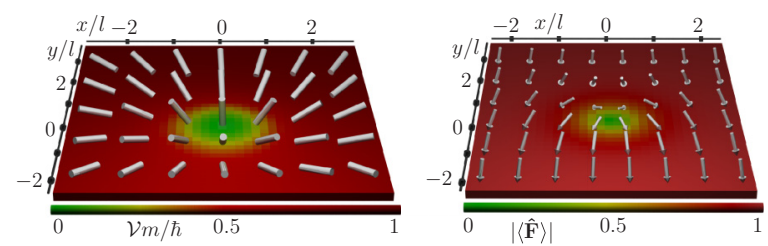

FIG. 5. Stable nematic coreless vortex in a BEC with polar interactions. (Left) The unoriented $\hat{\mathbf{d}}$ vector (cylinders) exhibits the coreless fountainlike texture. The circulation density $\mathcal{V}=\rho \mathbf{v} \cdot \hat{\boldsymbol{\varphi}}$ (color gradient) shows the composite-vortex structure, interpolating between the noncirculating polar phase to the outer singly quantized FM vortex. (Right) Corresponding spin texture $\langle\hat{\mathbf{F}}\rangle$ (arrows) and spin magnitude $|\langle\hat{\mathbf{F}}\rangle|$ (color gradient and arrow lengths), showing the core region. Conservation of magnetization forces the BEC into the FM phase away from the vortex line.

configuration at given $M$ can be qualitatively described by the spinor (44). However, our numerics demonstrate that the vortex is energetically stable only once magnetization is strong enough to deplete $\zeta_{+}$, enforcing an effective two-component regime. We find this to occur at $M \lesssim-0.2$. The stable vortex state shown in Fig. 5, then exhibits a Mermin-Ho-like texture in $\hat{\mathbf{d}}$ [corresponding to a charge $W=1 / 2$ as defined by Eq. (17) with $\hat{\mathbf{n}}_{F}=\hat{\mathbf{d}}$ ], and a corresponding bending of the spin vector from the $\hat{\boldsymbol{\rho}}$ direction at the center to the $-\hat{\mathbf{z}}$ direction in the FM region. The core size is again determined by the magnetization constraint. (We note in Sec. VII A that relaxing the energy of a singly quantized FM vortex also results in an effective two-component nematic coreless vortex when magnetization is sufficiently strong, demonstrating that the nematic coreless vortex can be stable also in the FM interaction regime.) The instability of the nematic coreless vortex at weaker magnetization results from the existence of lower-energy singular vortices with FM cores.

\section{SINGULAR VORTICES}

In Ref. [26] we calculated the core structures of energetically stable singly quantized vortices in the spin-1 BEC. In that analysis, the magnetization was allowed to vary during relaxation. We now calculate how these structures change if a weak longitudinal magnetization is preserved throughout the relaxation procedure. We find that the structure of the vortex cores is not notably affected unless the value of magnetization becomes very large. When the magnetization exceeds a critical value, the condensate relaxes to an effective two-component regime.

\section{A. Singular FM vortex}

For this analysis, it is instructive first to review the central features of the state that results from energy minimization if conservation of magnetization is not imposed. A trial wave function representing a singly quantized FM vortex is given by Eq. (18). The vortex is made up of overlapping vortex lines in the three spinor components, corresponding to a depletion of the atom density in the vortex core. The spin texture is uniform. As the energy is relaxed, these vortex lines move apart, such that the atom density is nonzero everywhere. The FM order 
parameter, however, remains singular on a well-defined vortex line, on which the atoms adopt the polar phase.

The size of a density-depleted vortex core would be determined by the density healing length $\xi_{n}$ [Eq. (4)]. By allowing the core to fill by perturbing $|\langle\hat{\mathbf{F}}\rangle|$, the core can expand to the size of the spin healing length $\xi_{F}$ [Eq. (4)], thereby lowering its energy. The deformation of the vortex core corresponds to a local rotation of the spin vector in an extended core region. The result is a spin winding around a core with nonvanishing density and $|\langle\hat{\mathbf{F}}\rangle|<1$. Away from the vortex core the initial uniform spin texture is preserved.

The stability of the singular FM vortex seems counterintuitive, since there also exists a stable coreless vortex with lower energy for the same parameters. However, in order for the singular vortex to decay into a coreless vortex with lower energy, the singular vortex would first have to exit the cloud (which requires a relatively slow rotation frequency), followed by nucleation of the coreless vortex (requiring sufficiently rapid rotation to overcome the energy barrier associated with vortex nucleation). The FM singly quantized vortex is therefore stable as a local energy minimum for a range of rotation frequencies.

In Ref. [26] we demonstrated how analysis and classification of the vortex-core structure is facilitated by a basis transformation to a natural spinor basis for the vortex state. In the absence of external magnetic fields, we are free to choose the spinor basis.

By transforming to the basis of spin projection onto the axis defined by the uniform $\langle\hat{\mathbf{F}}\rangle$ far from the vortex core, the spinor representing the relaxed core can be written in the form of an interpolation between an outer singular FM vortex and inner noncirculating polar phase, similar to Eq. (44). In this natural basis, the spinor reads

$$
\tilde{\zeta}=\frac{1}{2}\left(\begin{array}{c}
\sqrt{2} e^{i \varphi}\left(\cos ^{2} \frac{\tilde{\beta}}{2} D_{+}-\sin ^{2} \frac{\tilde{\beta}}{2} D_{-}\right) \\
\sin \tilde{\beta}\left(D_{+}+D_{-}\right) \\
\sqrt{2} e^{-i \varphi}\left(\sin ^{2} \frac{\tilde{\beta}}{2} D_{+}-\cos ^{2} \frac{\tilde{\beta}}{2} D_{-}\right)
\end{array}\right)
$$

where the angle $\tilde{\beta}(\rho)$ describes the tilt of the spin away from the new quantization axis. The vortex lines in $\widetilde{\zeta}_{ \pm}$overlap and the core is filled by $\tilde{\zeta}_{0}$. The interpolation between the FM vortex and the polar core is described by $D_{ \pm}(\rho)=\sqrt{1-F(\rho)}$ as defined in the construction of Eq. (30). The energetically stable vortex has the large-distance topology of a singular FM vortex, exhibiting a radial spin disgyration close to the vortex core. In the core of size $\sim \xi_{F}$, the topology represents the vortex-free polar phase.

In this natural basis we may define a magnetization

$$
\tilde{M}=\left(\tilde{N}_{+}-\tilde{N}_{-}\right) / N \text {, }
$$

where $\tilde{N}_{ \pm}$are the populations of the $\tilde{\zeta}_{ \pm}$spinor components. In the initial state, $\tilde{M}=1$. Owing to the rotation of the spin vector around the vortex core in the relaxed state, the contribution of the core region to the net magnetization is canceled out. Therefore, $\tilde{M}$ decreases during the relaxation. The spin then bends to point in the direction of the natural spinor basis over a non-negligible distance from the vortex core, leading to a further reduction in $\tilde{M}$. In the relaxed state, $\tilde{M} \simeq 0.5$ at the
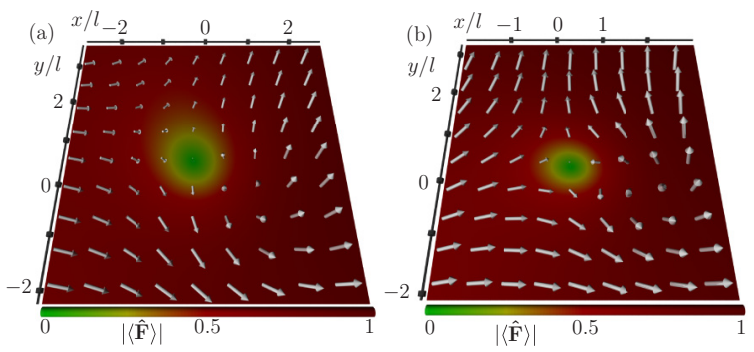

FIG. 6. Numerically calculated spin vector (arrows) and magnitudes (color gradient) for the singular vortex of the FM phase in the $x-y$ plane with a conserved magnetization of (a) $M=0.2$ and (b) $M=0.5$. Arrow lengths scale with the spin magnitude. The core structure remains as described in Ref. [26], with the conserved magnetization leading to a local rotation in spin space as the vortex core deforms.

lowest rotation frequency where the vortex becomes stable, increasing to $\tilde{M} \simeq 0.7$ at the upper limit of stability.

We are now in a position to understand how conserving an initial longitudinal magnetization $M$, defined by Eq. (3) in the basis of spin projection onto the $z$ axis, changes the relaxed state. The trial wave function for the singular FM vortex is again given by Eq. (18), where the constant angle $\beta_{0}$ is now chosen to yield the desired $M$. Energy relaxation under the condition that $M$ is conserved now results in the spin structure shown in Fig. 6. Again relaxation results in a local rotation of the spin vector to allow the vortex core to avoid the density depletion and instead fill with atoms with $|\langle\hat{\mathbf{F}}\rangle|<1$, expanding the size of the core to $\xi_{F}$, as illustrated in Fig. 6. However, when the magnetization is conserved, in addition to $\xi_{n}$ and $\xi_{F}$, also the magnetization length scale $\eta_{\mathrm{p}}$ given by Eq. (10) may affect the core structure. The length $\eta_{\mathrm{p}}$ defines the upper limit on the size of the non-FM core for any given $M$. As long as $M$ is sufficiently weak that $\eta_{\mathrm{p}} \gtrsim \xi_{F}$, the core size after energy relaxation is determined by $\xi_{F}$, and the filling of the vortex core may be understood from minimization of the gradient energy.

While the general understanding of the core-deformation mechanism is not qualitatively changed as long as $M$ is sufficiently weak, the resulting spin texture must adapt to the conserved magnetization. The general mechanism whereby the structure of the vortex core emerges as a result of the energetics associated with the two characteristic length scales $\xi_{n}$ and $\xi_{F}$ thus remains unaffected when conservation of magnetization is imposed. This description holds until $\xi_{F}>\eta_{\mathrm{p}}$, at which point the magnetization length constrains the core size.

Compared with the result found when not accounting for conservation of magnetization, the spins are everywhere tilted towards the $z$ axis. This compensates for the amount of magnetization that would otherwise be lost in the formation of the core region. Otherwise, the core structure remains qualitatively unchanged. Increasing $M$ leads to a further local rotation of the spin vector everywhere towards the $z$ axis, as shown in Fig. 6. We may then conclude that the effect of conserving a fixed longitudinal magnetization is to fix the natural basis of the vortex as long as $|M| \leqslant \tilde{M}$. Greater longitudinal magnetization cannot be achieved by tilting of the spin structure shown in Fig. 6. Our analysis thus immediately predicts a maximum magnetization $|M|=\tilde{M}$ above which the 
vortex state has to change, as stronger magnetization cannot be provided. At this magnetization strength, also $\eta_{\mathrm{p}} \sim \xi_{F}$ in our simulations, implying that the size of the vortex core becomes determined by the magnetization length scale rather than the spin healing length. At this point, magnetization causes depletion of the minority component, leading to an effective two-component vortex whose core region exhibits a nematic-coreless-vortex texture.

\section{B. Polar half-quantum vortex}

We now consider a condensate with a single half-quantum vortex. It is instructive first to ignore conservation of magnetization and analyze the resulting relaxed state. A trial wave function carrying a half-quantum vortex may be constructed from Eq. (25) by applying a spin rotation such that all spinor components have a nonzero population.

The trial wave function corresponds to a vortex where the atomic density vanishes on the singularity. The size of the core is then determined by the density healing length $\xi_{n}$ [Eq. (4)]. As energy relaxes, the vortex core is filled with atoms with $|\langle\hat{\mathbf{F}}\rangle|>0$, reaching the FM phase on the singularity of the polar order parameter. The vortex core can then expand to the size of the spin healing length $\xi_{F}$ [Eq. (4)]. In addition, a small region of nonzero $|\langle\hat{\mathbf{F}}\rangle|$ forms near the edge of the condensate, in which the spins antialign with the spin inside the vortex core. This effect appears counterintuitive, as exciting the wave function out of the polar phase costs interaction energy. This cost is, however, relatively small in the low-density region of the cloud and is offset by lowering the gradient energy arising from the filled vortex core.

Similarly to the analysis of the singular FM vortex, we may find a natural basis by transforming the wave function to the basis of spin quantization along the axis defined by the spin vector on the vortex line. The spinor then reads [cf. Eqs. (25) and (A2)]

$$
\tilde{\zeta}^{\mathrm{hq}}=\frac{1}{\sqrt{2}}\left(\begin{array}{c}
-\sqrt{1+\tilde{\mathcal{M}}(\rho)} \\
0 \\
e^{i \varphi} \sqrt{1-\tilde{\mathcal{M}}(\rho)}
\end{array}\right),
$$

where the local magnetization $\tilde{\mathcal{M}}$ describes the filling of the vortex core and the magnetization of the cloud edge. The largedistance topology of this vortex is that of the half-quantum vortex with nonzero magnetization (note that the FM phase is not observed far from the core) and the small-distance topology is that of the vortex-free FM phase.

With these observations in mind, we can now analyze the consequences of preserving a nonzero longitudinal magnetization of the vortex-carrying condensate. In order to give the trial wave function representing the half-quantum vortex a nonzero magnetization, we renormalize the occupations of the spinor components.

The relaxed half-quantum vortex state with fixed magnetization is shown in Fig. 7. On the vortex line, $|\langle\hat{\mathbf{F}}\rangle|=1$, and the core lowers its energy by expanding to the size allowed by the spin healing length. We also note that a significant region with nonzero $|\langle\hat{\mathbf{F}}\rangle|$ arises towards the edge of the cloud. From the spin texture we note that the longitudinal magnetization $M>0$ arises not from the vortex core, but from the magnetized edge regions. The spins in the edge regions remain nonzero to reduce
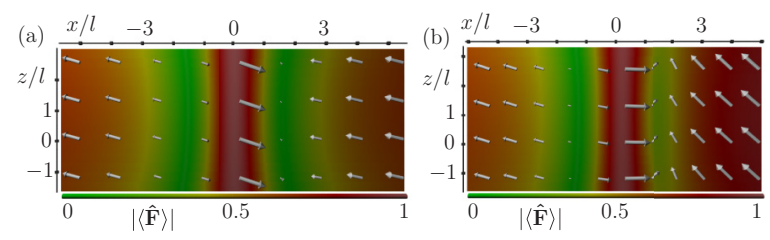

FIG. 7. Spin vector (arrows) and magnitude (color gradient) profiles in the $x-z$ plane for a half-quantum vortex with an initial magnetization $M=0.2$ which is (a) not conserved and (b) conserved. Arrow lengths scale with spin magnitude. The magnetization arises from the outer regions, not the FM core.

spin gradients. However, the spins in the edge regions no longer antialign with that inside the vortex core. This means that the treatment in terms of a natural spinor basis is no longer valid in a magnetized half-quantum vortex. The effect of fixing a weak longitudinal magnetization is to increase the magnitude of the spin in the outer region and to orient the spin in this region towards the direction of the applied magnetic field.

We find that magnetization of the edge region can only provide a total magnetization of $|M| \sim 0.3$, beyond which the vortex is no longer energetically stable. This is approximately the magnetization at which $\eta_{\mathrm{p}}<2 \xi_{F}$, at which point the halfquantum vortex carries a large gradient energy. However, the half-quantum vortex can be stabilized at greater magnetization, by a negative quadratic Zeeman splitting which is sufficiently strong to overcome the gradient energy. With $g_{2} B_{z}^{2}=-0.2 \hbar \omega$ in Eq. (1) the half-quantum vortex remains stable up to $|M| \sim$ 0.8 .

\section{Polar singly quantized vortex}

The energetic stability and structure of a singly quantized vortex in a polar spin-1 BEC were analyzed in detail in Ref. [26]. In that analysis, the initial state was entirely in the polar phase, and the magnetization was allowed to vary during energy relaxation. Energy minimization then resulted in a splitting of the singly quantized vortex into a pair of half-quantum vortices with FM cores, whose spins antialigned. In experiment, the core spin polarization was used to image the half-quantum vortices and thereby observe the splitting process [5]. This splitting preserves the overall topology of the initial state, but forms an extended core region where the phases mix. The filling of the vortex cores with atoms with $|\langle\hat{\mathbf{F}}\rangle| \geqslant 0$ and accommodating the singularities by requiring $|\langle\hat{\mathbf{F}}\rangle|=1$ on the singular lines lowers the total energy by reducing the gradient energies.

Similarly to the cloud with a single half-quantum vortex, the gradient energies associated with the vortex cores are again lowered by the formation of two magnetized edge regions, illustrated in Fig. 8(a). The spins in the two edge regions antialign with those in the nearest vortex core, immediately implying that the two edge regions exhibit spins pointing in opposite directions.

The formation of the magnetized edge regions is reminiscent of a composite topological defect such as those described by Eqs. (32) and (46). However, the breaking of axisymmetry renders the interpretation of this structure in terms of a hierarchical core structure prohibitively difficult. 

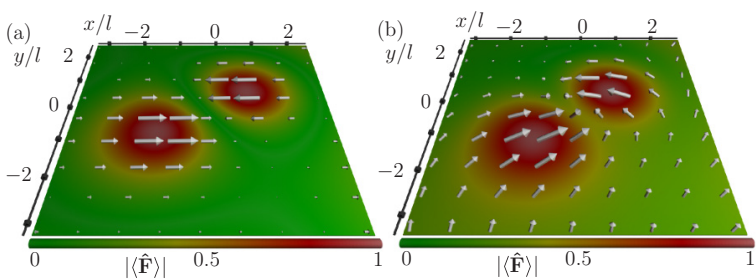

FIG. 8. Numerically calculated spin magnitude (color gradient) and vector (arrows) profiles of the singular polar vortex in the $x-y$ plane when an initial magnetization $M=0.2$ is (a) not conserved and (b) conserved. Arrow lengths scale with spin magnitude. The singular vortex deforms to a pair of half-quantum vortices with FM cores in both cases.

This picture remains unchanged as we account for a conserved magnetization of zero. This is to be expected as the structure described above has zero net magnetization. However, a conserved, weak, nonzero magnetization does modify the structure somewhat. A magnetized singly quantized vortex can be constructed from Eq. (23) by adjusting the populations of the spinor components to give the desired magnetization. We now explicitly conserve this magnetization throughout energy relaxation. The resulting relaxed states for conserved and nonconserved weak, nonzero magnetization are shown in Fig. 8, and we note the splitting of the initial vortex into two singular lines in both cases, which may be identified as half-quantum vortices.

In contrast to the isolated half-quantum vortex, the nonzero magnetization $M$ of the condensate is carried by the vortex cores rather than the edge regions. When the condensate contained only a single half-quantum vortex, the spin structure could adjust to a varying magnetization $M$ by a simple rotation of the spins everywhere in the cloud. Because the spins in the two edge regions antialign when magnetization is not conserved, an equal rotation of spins everywhere can no longer increase the longitudinal magnetization. Therefore, the effect of preserving a nonzero $M$ throughout energy relaxation is to cause the spins in the two vortex cores to orient differently so that they no longer antialign. This provides the required net magnetization. The gradient energy in the extended core region is reduced by this process and so the magnitude of the spin in the edge regions is not required to be as strong as in the case of zero magnetization.

Increasing the magnetization causes the spins in the magnetized cores to orient toward the direction of the applied magnetic field. The cores also draw closer together. Further increasing the magnetization leads to the nonpolar regions overlapping and forming a single core with a continuous spin texture, exhibiting the fountain structure associated with the coreless vortex. Coexistence of different core symmetries of the singly quantized polar vortex is explored in Ref. [86]. There it is shown how the core isotropy is sensitive to tunable Zeeman shifts, which can continuously restore the axial symmetry. Here we explain how the vortex core structure arises from the magnetization constraint that the FM core of the vortex line can provide.

The core regions can only provide a weak magnetization as their size is constrained by the spin healing length. The magnetization length scale $\eta_{\mathrm{f}}$ given by Eq. (8) described the smallest core size required to yield a given magnetization $M$. Hence, when $M$ is sufficiently strong that $\eta_{\mathrm{f}} \gtrsim \xi_{F}$, the magnetization cannot be upheld by the vortex cores alone, leading to energetic instability of the state in Fig. 8. This leads to depletion of the minority component, resulting in a two-component coreless vortex similar to Fig. 3. We find that this happens at $|M| \sim 0.3$. While a negative quadratic Zeeman splitting could restabilize a single half-quantum vortex at higher magnetization, the same is not true for the split singly quantized vortex.

In conclusion, we find that also in the polar regime, the results of Ref. [26] remain qualitatively unchanged when accounting for conservation of a sufficiently weak initial magnetization, exhibiting the splitting of the vortex core. However, the required magnetization in the relaxed state is produced by forcing the resulting FM vortex cores to no longer exhibit anti-aligning spins. As magnetization increases, the vortex cores gradually merge, eventually exhibiting an effective two-component coreless vortex as $|M| \gtrsim 0.3$. Hence, in the polar interaction regime, initial coreless and singly quantized vortices relax to the same defect structure at both weak and strong magnetization.

\section{EXAMPLES OF COMPLEX VORTEX-CORE HIERARCHIES}

In this section we consider the more complex composite vortices implied by the solutions in Table I that connect singular vortex states in both their FM and polar limits. In these cases a more complex hierarchy could form, exhibiting several concentric core regions where the phases alternate as the inner singular vortex itself develops a filled core. These highly complex core structures are not, in general, energetically stable. However, we demonstrate here how FM vortices with polar half-quantum or singly quantized vortex small-distance topology may be stabilized.

\section{A. Half-quantum vortex core of FM vortex}

In Table I, we presented analytic vortex solutions representing composite topological defects, including the nontrivial case of a singular polar vortex forming the core of an outer singular FM vortex. [See also Eq. (A2).] As energy relaxes, a three-step vortex hierarchy may form: The largedistance topology represents the singular FM vortex with spin $\langle\hat{\mathbf{F}}\rangle=\sin \beta \hat{\mathbf{x}}+\cos \beta \hat{\mathbf{z}}$. Inside its core, $F(\rho)$ decreases to 0 , displaying a half-quantum vortex with $\hat{\mathbf{d}}=\cos \beta \cos (\varphi / 2) \hat{\mathbf{x}}+$ $\sin (\varphi / 2) \hat{\mathbf{y}}-\sin \beta \cos (\varphi / 2) \hat{\mathbf{z}}$. To avoid depletion of the atom density, $F(\rho)$ may then increase back to $F(\rho \rightarrow 0)=1$ inside the core of the half-quantum vortex, corresponding to the vortex-free FM phase. A similar composite vortex state was considered in a recent topological classifications of vortex cores in spin-1 BECs [27].

We now ask whether the composite-vortex structure can form as the energy of the singular FM vortex relaxes and whether it can be energetically stable. We consider again the trial wave function for a singly quantized FM vortex, constructed from Eq. (18) with magnetization $0 \leqslant M \leqslant 0.8$, in a condensate with FM interactions. We find that in order 
for the composite-vortex structure to replace the vortex-free polar core in the stable state, a sufficiently strong negative quadratic Zeeman splitting is required. This can be induced by combining a static magnetic field with a microwave dressing field, generating and ac Stark shift that corresponds to a highly tunable quadratic level shift [87]. The level shift may also be induced by lasers [88]. Here we take $g_{2} B_{z}^{2}=-0.2 \hbar \omega$. The negative quadratic Zeeman effect favors occupation of the $m= \pm 1$ Zeeman levels. This causes the spin vector to align (antialign) with the $z$ axis away from the vortex, and to antialign (align) with it in the FM core. The two possible spin alignments are energetically degenerate (though conservation of magnetization may only allow one).

The quadratic Zeeman splitting required to energetically stabilize the composite-vortex structure is strong enough that $\beta$ is forced to adopt values of either 0 or $\pi$, and the $\zeta_{0}$ spinor component is empty. In the resulting effective two-component limit, the spinor can be parametrized as

$$
\zeta^{\mathrm{c}}=\frac{1}{\sqrt{2}}\left(\begin{array}{c}
-e^{i \varphi} \sqrt{1+F_{z}(\rho)} \\
0 \\
\sqrt{1-F_{z}(\rho)}
\end{array}\right)
$$

where $\langle\hat{\mathbf{F}}\rangle=F_{z} \hat{\mathbf{z}}$, with $F_{z}=-1$ in the inner core and $F_{z}=$ 1 away from the vortex. It is now readily apparent that the vortex line in $\zeta_{+}^{c}$ represents the overall topology of the singly quantized FM vortex at sufficiently large $\rho$. Similarly, in the inner core, $\zeta_{-}^{\mathrm{c}}$ represents a vortex-free FM wave function. Where $F_{z} \rightarrow 0$ in the intermediate region, $\zeta^{\mathrm{c}}$ takes the form of a half-quantum vortex similar to Eq. (25).

Due to the FM interaction, the thickness of the polar region is restricted by $\xi_{F}$. Hence, the polar vortex takes on the character of a domain wall separating an outer spin domain from an inner domain with opposing spin. The size of the inner FM core is not constrained by the spin healing length, as it does not violate the FM spin condition.

However, in the atomic spinor BEC, magnetization is conserved. The effect of this is to determine the size of the inner FM core, so that the antialigned spins in the FM regions yield the required magnetization. This corresponds to a length scale $\eta_{\mathrm{d}}$ associated with the conserved magnetization. In a simplified model that ignores the thickness of the domain wall, an estimate for $\eta_{\mathrm{d}} \simeq R_{\mathrm{TF}} \sqrt{1-[(1+M) / 2]^{2 / 5}}$ may be derived analogously to the magnetization length scales defined in Sec. II. From this we can understand the upper limit on magnetization for which the composite vortex is stable: The magnetization must not cause the gradient energies associated with a small core to overcome the Zeeman energy. This happens when $\eta_{\mathrm{d}} \lesssim \xi_{Z}=l\left(\hbar \omega / 2\left|g_{2}\right| B_{z}^{2}\right)^{1 / 2}$, the healing length associated with the quadratic Zeeman energy, in agreement with our numerical results. The vortex structure at $M=0.6$ is shown in Fig. 9, demonstrating the formation of the composite core in which the innermost region exhibits the noncirculating FM phase.

It is interesting to note that the conservation of a nonzero magnetization is required to stabilize this vortex in the FM regime. If the magnetization is not conserved, or is conserved at zero, the gradient energy associated with the domain wall renders the vortex unstable.
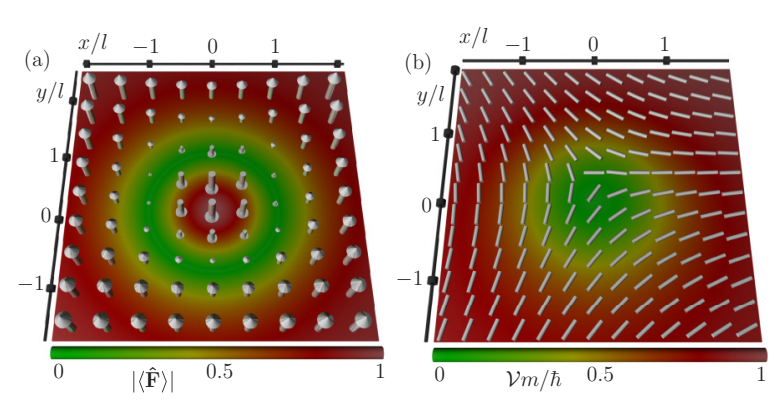

FIG. 9. (a) Numerically calculated spin texture (arrows) and magnitude (color gradient) in the $x-y$ plane for the composite vortex in the FM regime with $M=0.6$. Arrow lengths scale with spin magnitude. (b) Nematic axis profile (cylinders) and circulation (color gradient) for the same, showing the noncirculating inner FM core and winding of $\hat{\mathbf{d}}$ by $\pi$.

A sufficiently strong magnetization $|M| \gtrsim 0.3$ together with the quadratic Zeeman energy also allows the compositevortex structure to be energetically stable in a BEC with polar interactions. The strong magnetization is upheld by forcing the condensate away from the vortex line towards the FM phase, forming a singular vortex. Again the required Zeeman energy causes depopulation of $\zeta_{0}$. In contrast to the FM regime, the polar interactions now imply that that size of the FM core is determined by the spin healing length, while the thickness of the polar region is determined by the magnetization. The quadratic Zeeman splitting is able to stabilize the composite vortex provided $\eta_{\mathrm{d}}>\xi_{Z}$, corresponding to magnetization of $M \sim 0.9$, which is supported by our numerics. When magnetization is not conserved, both the half-quantum and singly quantized vortices of the polar phase described in Sec. VII B are stable under the influence of quadratic Zeeman splitting. For magnetization $M<0.3$, the half-quantum vortex remains stable. The stronger magnetization serves to increase $|\langle\hat{\mathbf{F}}\rangle|$ near the edge of the trap, forming the FM phase.

\section{B. Singly quantized vortex core of FM vortex}

Inspired by the results described in Fig. 9 and the observations made in Sec. VI A 2, we can envisage using a designed quadratic Zeeman shift to stabilize complex composite defect structures by locally forcing the condensate into the polar phase. A highly tunable quadratic level shift can in principle be imposed using lasers [88]. Consider, e.g., the spatial profile of a Laguerre-Gaussian beam,

$$
q=q_{0}\left(\frac{2 \rho^{2}}{w^{4}}\right)^{m} e^{-2 \rho^{2} / w^{2}}\left[L_{n}^{m}\left(\frac{2 \rho^{2}}{w^{2}}\right)\right]^{2},
$$

where $m$ and $n$ give the Laguerre-Gaussian modes and $w$ is the beam-waist parameter. If a quadratic Zeeman shift is induced using Eq. (54), concentric regions can be created where the polar (FM) phase is favored by the presence (absence) of the level shift. Using high Laguerre-Gaussian modes, these regions may be made quite sharp.

As an illustration of a composite vortex structure stabilized this way, we consider a FM condensate containing a phaseimprinted singular vortex at $M=0$. This is allowed to relax, conserving $M$ throughout, in the presence of a positive 


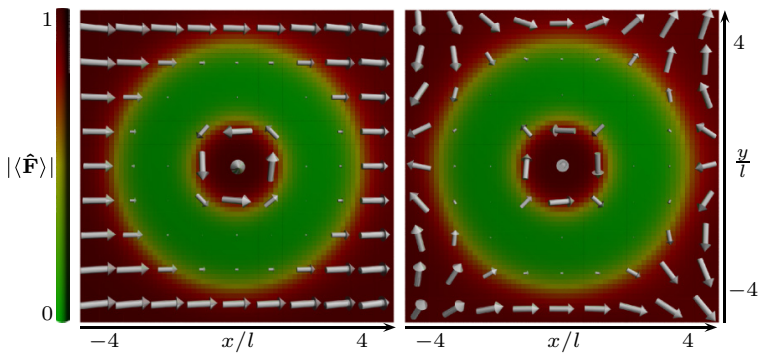

FIG. 10. Spin magnitude (color scale) and spin vector (arrows) showing composite vortex states arising as a singly quantized vortex in a FM condensate relaxes in the presence of an engineered quadratic Zeeman shift with Laguerre-Gaussian profile that forces the condensate into the polar phase in an annulus surrounding the vortex core. (Left) Trap rotation $\Omega=0.125 \omega$. A coreless vortex appears in the inner FM core, surrounded by a singly quantized polar vortex, together forming the extended core of a singular FM vortex. (Right) $\Omega=0.135 \omega$. Rotation causes a multiply quantized vortex to form in the outer FM region.

quadratic Zeeman shift with $(m, n)=(6,0)$ Laguerre-Gaussian profile. (In order to more easily produce a sharply defined vortex structure we assume $N c_{0}=10^{4} \hbar \omega l^{3}$ together with an artificially strong FM interaction $N c_{2}=463 \hbar \omega l^{3}$.) The resulting vortex state is shown in Fig. 10 for two different trap-rotation frequencies. The positive quadratic Zeeman shift favors the $\zeta_{0}$ component and locally forces the condensate into the polar phase in a ring-shaped region. The relaxed vortex state exhibits a concentric core structure, where an inner FM coreless vortex is surrounded by a singly quantized polar vortex, which in turn is surrounded by the singly quantized FM vortex far away from the vortex line. If rotation is increased, the part of the cloud corresponding to the large-distance topology may exhibit a multiply quantized vortex. Such a solution is shown to the right in Fig. 10.

\section{CONCLUDING REMARKS}

On time scales where $s$-wave scattering dominates the interactions in a spin-1 BEC, the longitudinal magnetization $M=\left(N_{+}-N_{-}\right) / N$ is approximately conserved [56]. (Magnetization may change on longer time scales due to, for example, dipolar interactions, atom loss, or spurious $p$-wave scattering with high-temperature atoms.) We have determined the structure and stability of singular and nonsingular vortices by numerically relaxing the energy of trial wave functions, explicitly imposing conservation of magnetization throughout the relaxation procedure. In order to describe vortex states that mix FM and polar phases, we have analytically constructed spinor wave functions for vortices that smoothly interpolate between the two manifolds. In particular, we have explicitly derived solutions that describe the filled cores of singular vortices. Such filling of the vortex core occurs very generally in the spinor BECs as result of energetic competition between characteristic length scales as the energy relaxes. We have also provided analytic construction of solutions that represent composite defects where both polar and FM limits correspond to vortex states. These exhibit different vortex topology on small and large distances from the vortex line. An example of a stable composite defect is a FM coreless vortex in the polar interaction regime [34]. In addition to composite defects occurring purely as a result of conservation of magnetization, we have suggested how vortices with a concentric hierarchy of cores may be stabilized in experiment.

\section{ACKNOWLEDGMENTS}

We acknowledge financial support from the EPSRC and the Leverhulme Trust, as well as the use of the IRIDIS High Performance Computing Facility at the University of Southampton.

\section{APPENDIX: ANALYTIC WAVE FUNCTIONS FOR FM VORTICES WITH POLAR HALF-QUANTUM VORTEX CORE}

Just as the singly quantized polar vortex may form inside the core of a singular FM vortex (or vice versa), a polar half-quantum vortex may form inside the singular FM vortex core (and vice versa), as demonstrated in Sec. VIII. Here we construct an explicit spinor for this composite vortex state by requiring $\tau=-\gamma=\varphi / 2$ and $\alpha=0$. Taking constant $\beta=\beta_{0}$, Eq. (30) then becomes

$$
\zeta=\frac{\mathcal{E}}{2}\left(\begin{array}{c}
\sqrt{2}\left(\mathcal{E}^{-1} \sin ^{2} \frac{\beta_{0}}{2} D_{-}-\mathcal{E} \cos ^{2} \frac{\beta_{0}}{2} D_{+}\right) \\
-\sin \beta_{0}\left(\mathcal{E}^{-1} D_{-}+\mathcal{E} D_{+}\right) \\
\sqrt{2}\left(\mathcal{E}^{-1} \cos ^{2} \frac{\beta_{0}}{2} D_{-}-\mathcal{E} \sin ^{2} \frac{\beta_{0}}{2} D_{+}\right)
\end{array}\right),
$$

where $\mathcal{E}=\exp (i \varphi / 2)$. In the $F=1$ limit we recover the singular FM vortex (18) with uniform spin profile $\langle\hat{\mathbf{F}}\rangle=$ $\sin \beta_{0} \hat{\mathbf{x}}+\cos \beta_{0} \hat{\mathbf{z}}$. The $F=0$ limit, on the other hand, is a halfquantum vortex with $\hat{\mathbf{d}}=\cos \beta_{0} \cos (\varphi / 2) \hat{\mathbf{x}}-\sin (\varphi / 2) \hat{\mathbf{y}}-$ $\sin \beta_{0} \cos (\varphi / 2) \hat{\mathbf{z}}$ exhibiting the characteristic $\pi$ winding as the vortex line is encircled. Hence, the half-quantum vortex forms the core of the FM singular vortex when $F(\rho)$ increases from $F=0$ to $F=1$ with the radial distance. Correspondingly, the circulation $v=h(1+F) / 2 m$ interpolates smoothly between the inner half-quantum of circulation and the outer singly quantized FM vortex.

The FM order parameter also allows the formation of a singular vortex on the form of Eq. (19), which is topologically equivalent to Eq. (18), and the one may be deformed into the other by local spin rotations. We should therefore expect also the core of Eq. (19) to be able to host a polar half-quantum vortex. We find an expression for this composite vortex state by taking $\tau=\gamma=\varphi / 2, \alpha=\varphi$. Then

$$
\zeta=\frac{\mathcal{E}}{2}\left(\begin{array}{c}
\sqrt{2}\left[\mathcal{E}^{-1} \sin ^{2} \frac{\beta(\rho)}{2} D_{-}-\mathcal{E}^{-3} \cos ^{2} \frac{\beta(\rho)}{2} D_{+}\right] \\
-\sin \beta(\rho)\left(\mathcal{E} D_{-}+\mathcal{E}^{-1} D_{+}\right) \\
\sqrt{2}\left[\mathcal{E}^{3} \cos ^{2} \frac{\beta(\rho)}{2} D_{-}-\mathcal{E} \sin ^{2} \frac{\beta(\rho)}{2} D_{+}\right]
\end{array}\right) .
$$

The $F=1$ limit represents the FM singular vortex (19), exhibiting the radial spin disgyration $\langle\hat{\mathbf{F}}\rangle=\sin \beta(\rho) \hat{\boldsymbol{\rho}}+\cos \beta(\rho) \hat{\mathbf{z}}$, by construction. The angle $\beta(\rho)$ increases from $\pi / 2$ to $\pi$ as a function of radius. In the $F=0$ limit, this spinor does indeed represent a half-quantum vortex with $\tau$ winding by $\pi$. The winding of $\hat{\mathbf{d}}$ depends on $\beta$, resulting in a nematic axis profile of $\hat{\mathbf{d}}=[\cos \varphi \cos \varphi / 2 \cos \beta(\rho)-$ $\sin \varphi \sin \varphi / 2] \hat{\mathbf{x}}+[\sin \varphi \cos \varphi / 2 \cos \beta(\rho)+\cos \varphi \sin \varphi / 2] \hat{\mathbf{y}}-$ 
$\cos \varphi / 2 \sin \beta(\rho) \hat{\mathbf{z}}$. Again the composite defect is formed as $F(\rho)$ is allowed to vary such that $F(\rho \rightarrow 0)=0$ and $F(\rho)=1$ at large $\rho$, and the circulation
$v=h[1-F-2 F \cos \beta(\rho)] / 2 m$ interpolates from the inner half-quantum vortex of the polar phase to the nonquantized circulation of the outer radial spin disgyration.
[1] D. M. Stamper-Kurn, M. R. Andrews, A. P. Chikkatur, S. Inouye, H.-J. Miesner, J. Stenger, and W. Ketterle, Phys. Rev. Lett. 80, 2027 (1998).

[2] J. Stenger, S. Inouye, D. M. Stamper-Kurn, H. Miesner, A. P. Chikkatur, and W. Ketterle, Nature (London) 396, 345 (1998).

[3] M. W. Ray, E. Ruokokoski, S. Kandel, M. Möttönen, and D. S. Hall, Nature (London) 505, 657 (2014).

[4] M. W. Ray, E. Ruokokoski, K. Tiurev, M. Möttönen, and D. S. Hall, Science 348, 544 (2015).

[5] S. W. Seo, S. Kang, W. J. Kwon, and Y.-i. Shin, Phys. Rev. Lett. 115, 015301 (2015).

[6] A. E. Leanhardt, Y. Shin, D. Kielpinski, D. E. Pritchard, and W. Ketterle, Phys. Rev. Lett. 90, 140403 (2003).

[7] L. S. Leslie, A. Hansen, K. C. Wright, B. M. Deutsch, and N. P. Bigelow, Phys. Rev. Lett. 103, 250401 (2009).

[8] J.-Y. Choi, W. J. Kwon, and Y.-I. Shin, Phys. Rev. Lett. 108, 035301 (2012).

[9] J.-y. Choi, W. J. Kwon, M. Lee, H. Jeong, K. An, and Y.-i. Shin, New J. Phys. 14, 053013 (2012).

[10] L. E. Sadler, J. M. Higbie, S. R. Leslie, M. Vengalattore, and D. M. Stamper-Kurn, Nature (London) 443, 312 (2006).

[11] M. Vengalattore, S. R. Leslie, J. Guzman, and D. M. StamperKurn, Phys. Rev. Lett. 100, 170403 (2008).

[12] J. Kronjäger, C. Becker, P. Soltan-Panahi, K. Bongs, and K. Sengstock, Phys. Rev. Lett. 105, 090402 (2010).

[13] Y. Kawaguchi and M. Ueda, Phys. Rep. 520, 253 (2012).

[14] S.-K. Yip, Phys. Rev. Lett. 83, 4677 (1999).

[15] U. Leonhardt and G. Volovik, JETP Lett. 72, 46 (2000).

[16] T. Isoshima and K. Machida, Phys. Rev. A 66, 023602 (2002).

[17] T. Mizushima, K. Machida, and T. Kita, Phys. Rev. A 66, 053610 (2002).

[18] F. Zhou, Int. J. Mod. Phys. B 17, 2643 (2003).

[19] H. Saito, Y. Kawaguchi, and M. Ueda, Phys. Rev. Lett. 96, 065302 (2006).

[20] L. Santos and T. Pfau, Phys. Rev. Lett. 96, 190404 (2006).

[21] G. W. Semenoff and F. Zhou, Phys. Rev. Lett. 98, 100401 (2007).

[22] R. Barnett, A. Turner, and E. Demler, Phys. Rev. A 76, 013605 (2007).

[23] A.-C. Ji, W. M. Liu, J. L. Song, and F. Zhou, Phys. Rev. Lett. 101, 010402 (2008).

[24] M. Kobayashi, Y. Kawaguchi, M. Nitta, and M. Ueda, Phys. Rev. Lett. 103, 115301 (2009).

[25] J. A. M. Huhtamäki, T. P. Simula, M. Kobayashi, and K. Machida, Phys. Rev. A 80, 051601 (2009).

[26] J. Lovegrove, M. O. Borgh, and J. Ruostekoski, Phys. Rev. A 86, 013613 (2012).

[27] S. Kobayashi, Y. Kawaguchi, M. Nitta, and M. Ueda, Phys. Rev. A 86, 023612 (2012).

[28] T. Mawson, G. Ruben, and T. Simula, Phys. Rev. A 91, 063630 (2015).

[29] T. Mizushima, K. Machida, and T. Kita, Phys. Rev. Lett. 89, 030401 (2002).

[30] J.-P. Martikainen, A. Collin, and K.-A. Suominen, Phys. Rev. A 66, 053604 (2002).
[31] E. J. Mueller, Phys. Rev. A 69, 033606 (2004).

[32] J. W. Reijnders, F. J. M. van Lankvelt, K. Schoutens, and N. Read, Phys. Rev. A 69, 023612 (2004).

[33] M. Takahashi, V. Pietilä, M. Möttönen, T. Mizushima, and K. Machida, Phys. Rev. A 79, 023618 (2009).

[34] J. Lovegrove, M. O. Borgh, and J. Ruostekoski, Phys. Rev. Lett. 112, 075301 (2014).

[35] H. T. C. Stoof, E. Vliegen, and U. Al Khawaja, Phys. Rev. Lett. 87, 120407 (2001).

[36] J. Ruostekoski and J. R. Anglin, Phys. Rev. Lett. 91, 190402 (2003).

[37] C. M. Savage and J. Ruostekoski, Phys. Rev. A 68, 043604 (2003).

[38] V. Pietilä and M. Möttönen, Phys. Rev. Lett. 103, 030401 (2009).

[39] E. Ruokokoski, V. Pietilä, and M. Möttönen, Phys. Rev. A 84, 063627 (2011).

[40] M. O. Borgh and J. Ruostekoski, Phys. Rev. Lett. 109, 015302 (2012).

[41] M. O. Borgh, J. Lovegrove, and J. Ruostekoski, New J. Phys. 16, 053046 (2014).

[42] J. Ruostekoski and J. R. Anglin, Phys. Rev. Lett. 86, 3934 (2001).

[43] U. Al Khawaja and H. Stoof, Nature (London) 411, 918 (2001).

[44] R. A. Battye, N. R. Cooper, and P. M. Sutcliffe, Phys. Rev. Lett. 88, 080401 (2002).

[45] C. M. Savage and J. Ruostekoski, Phys. Rev. Lett. 91, 010403 (2003).

[46] K. Kasamatsu, M. Tsubota, and M. Ueda, Phys. Rev. Lett. 91, 150406 (2003).

[47] J. Ruostekoski, Phys. Rev. A 70, 041601 (2004).

[48] H. Takeuchi and M. Tsubota, J. Phys. Soc. Jpn, 75, 063601 (2006).

[49] K. Kasamatsu, H. Takeuchi, M. Nitta, and M. Tsubota, J. High Energy Phys. 011 (2010) 068.

[50] P. Mason and A. Aftalion, Phys. Rev. A 84, 033611 (2011).

[51] T. Kawakami, T. Mizushima, M. Nitta, and K. Machida, Phys. Rev. Lett. 109, 015301 (2012).

[52] M. Nitta, K. Kasamatsu, M. Tsubota, and H. Takeuchi, Phys. Rev. A 85, 053639 (2012).

[53] W. Zhang, S. Yi, and L. You, New J. Phys. 5, 77 (2003).

[54] M.-S. Chang, C. D. Hamley, M. D. Barrett, J. A. Sauer, K. M. Fortier, W. Zhang, L. You, and M. S. Chapman, Phys. Rev. Lett. 92, 140403 (2004).

[55] H. Mäkelä and K.-A. Suominen, Phys. Rev. A 75, 033610 (2007).

[56] D. Jacob, L. Shao, V. Corre, T. Zibold, L. De Sarlo, E. Mimoun, J. Dalibard, and F. Gerbier, Phys. Rev. A 86, 061601 (2012).

[57] D. M. Stamper-Kurn and M. Ueda, Rev. Mod. Phys. 85, 1191 (2013).

[58] J. Javanainen and J. Ruostekoski, J. Phys. A: Mat. Gen. 39, L179 (2006).

[59] E. G. M. van Kempen, S. J. J. M. F. Kokkelmans, D. J. Heinzen, and B. J. Verhaar, Phys. Rev. Lett. 88, 093201 (2002). 
[60] S. Knoop, T. Schuster, R. Scelle, A. Trautmann, J. Appmeier, M. K. Oberthaler, E. Tiesinga, and E. Tiemann, Phys. Rev. A 83, 042704 (2011).

[61] M. O. Borgh and J. Ruostekoski, Phys. Rev. A 87, 033617 (2013).

[62] N. D. Mermin, Rev. Mod. Phys. 51, 591 (1979).

[63] V. R. Chechetkin, Zh. Eksp. Teor. Fiz. 71, 1463 (1976) [JETP 44, 766 (1976)].

[64] P. W. Anderson and G. Toulouse, Phys. Rev. Lett. 38, 508 (1977).

[65] N. D. Mermin and T.-L. Ho, Phys. Rev. Lett. 36, 594 (1976).

[66] N. Manton and P. Sutcliffe, Topological Solitons (Cambridge University Press, Cambridge, UK, 2004).

[67] T. H. R. Skyrme, Proc. R. Soc. London, Ser. A 260, 127 (1961).

[68] M. M. Salomaa and G. E. Volovik, Phys. Rev. Lett. 55, 1184 (1985).

[69] M. M. Salomaa and G. E. Volovik, Rev. Mod. Phys. 59, 533 (1987).

[70] P. G. de Gennes and J. Prost, The Physics of Liquid Crystals, 2nd ed. (Oxford University Press, Oxford, U.K., 1993).

[71] K.-P. Marzlin, W. Zhang, and E. M. Wright, Phys. Rev. Lett. 79, 4728 (1997).

[72] E. L. Bolda and D. F. Walls, Phys. Lett. A 246, 32 (1998).

[73] J. E. Williams and M. J. Holland, Nature (London) 401, 568 (1999).

[74] M. Nakahara, T. Isoshima, K. Machida, S. Ichiro Ogawa, and T. Ohmi, Phys. B (Amsterdam, Neth.) 284, 17 (2000).

[75] T. Isoshima, M. Nakahara, T. Ohmi, and K. Machida, Phys. Rev. A 61, 063610 (2000).
[76] Z. Dutton and J. Ruostekoski, Phys. Rev. Lett. 93, 193602 (2004).

[77] M. R. Matthews, B. P. Anderson, P. C. Haljan, D. S. Hall, C. E. Wieman, and E. A. Cornell, Phys. Rev. Lett. 83, 2498 (1999).

[78] A. E. Leanhardt, A. Görlitz, A. P. Chikkatur, D. Kielpinski, Y. Shin, D. E. Pritchard, and W. Ketterle, Phys. Rev. Lett. 89, 190403 (2002).

[79] Y. Shin, M. Saba, M. Vengalattore, T. A. Pasquini, C. Sanner, A. E. Leanhardt, M. Prentiss, D. E. Pritchard, and W. Ketterle, Phys. Rev. Lett. 93, 160406 (2004).

[80] M. F. Andersen, C. Ryu, P. Cladé, V. Natarajan, A. Vaziri, K. Helmerson, and W. D. Phillips, Phys. Rev. Lett. 97, 170406 (2006).

[81] R. Pugatch, M. Shuker, O. Firstenberg, A. Ron, and N. Davidson, Phys. Rev. Lett. 98, 203601 (2007).

[82] The experiment in Ref. [9] actually starts from $\zeta=(0,0,1)^{T}$ and creates an "upside-down" coreless vortex. .

[83] K. Murata, H. Saito, and M. Ueda, Phys. Rev. A 75, 013607 (2007).

[84] J. Ruostekoski and Z. Dutton, Phys. Rev. A 76, 063607 (2007).

[85] C. Pethick and H. Smith, Bose-Einstein Condensation in Dilute Gases (Cambridge University Press, Cambridge, U.K., 2002).

[86] M. O. Borgh, M. Nitta, and J. Ruostekoski, Phys. Rev. Lett. 116, 085301 (2016).

[87] F. Gerbier, A. Widera, S. Fölling, O. Mandel, and I. Bloch, Phys. Rev. A 73, 041602 (2006).

[88] L. Santos, M. Fattori, J. Stuhler, and T. Pfau, Phys. Rev. A 75, 053606 (2007). 